\title{
Flow around an oscillating grid in water and shear-thinning polymer solution at low Reynolds number
}

\author{
Tom Lacassagne $^{1,2}\left(\mathbb{D} \cdot\right.$ Adrien Lyon $^{1} \cdot$ Serge Simoëns ${ }^{1} \cdot$ Mahmoud El Hajem $^{1} \cdot$ Jean-Yves Champagne ${ }^{1}$
}

Received: 8 July 2019 / Revised: 23 October 2019 / Accepted: 29 October 2019 / Published online: 3 December 2019

(C) The Author(s) 2019

\begin{abstract}
The study of turbulence in complex fluids is of great interest in many environmental and industrial applications, in which the interactions between liquid phase rheology, turbulence, and other phenomena such as mixing or heat and mass transfer have to be understood. Oscillating grid stirred tanks have been used for many purposes in research involving turbulence. However, the mechanisms of turbulence production by the oscillating grid itself have never been studied, and oscillating grid turbulence (OGT) remained undescribed in non-Newtonian, shear-thinning, dilute polymer solutions until recently (Lacassagne et al., in Phys Fluids 31(8):083,102, 2019). The aim of this paper is to study the influence of the shear-thinning property of dilute polymer solutions (DPS), such as xanthan gum (XG), on mean flow, oscillatory flows, and turbulence around an oscillating grid. Liquid phase velocity is measured by particle image velocimetry (PIV) in a vertical plane above the central grid bar. Mean, oscillatory and turbulent components of the velocity fields are deduced by triple Hussain-Reynolds decomposition based on grid phase-resolved measurements. Outside of the grid swept region, the amplitude of oscillatory fluctuations quickly become negligible compared to that of turbulent fluctuations, and the triple and classical Reynolds decomposition become equivalent. Oscillatory jets and wakes behind the grid and their interactions are visualized. Turbulent (Reynolds) and oscillatory stresses are used to evidence a modification of oscillatory flow and turbulence intensity repartition in and around the grid swept region. Energy transfer terms between mean, oscillatory and turbulent flows are estimated and used to describe turbulence production in the grid swept region. Energy is injected by the grid into the oscillatory component. In water, it is transferred to turbulence mostly inside the grid swept region. In DPS, oscillatory flow persists outside of the grid swept zone. Energy is transferred not only to turbulence, in the grid swept region, and far from the tank's walls, but also to the mean flow, leading to an enhancement of the latter. Mean flow production and enhancement mechanisms are explainable by oscillatory jet variable symmetry and intensity, and by time- and space-variable viscosity. Backward transfer from turbulence to oscillatory flow is also evidenced in DPS. Finally, using phased root mean square (rms) values of turbulent velocity fluctuations, it is shown that in water, the decay of turbulence intensity behind an oscillating grid can be related to the decay of fixed grid turbulence for specific grid positions, a result no longer valid in DPS.
\end{abstract}

Tom Lacassagne

tom.lacassagne@gmail.com

Serge Simoëns

serge.simoens@ec-lyon.fr

1 Univ Lyon, INSA de Lyon, Ecole Centrale de Lyon, Université Lyon 1, CNRS, LMFA UMR 5509, 69621 Villeurbanne Cedex, France

2 Department of Mechanical Engineering, University College London, Torrington Place, London WC1E 6BT, UK 


\section{Graphic abstract}

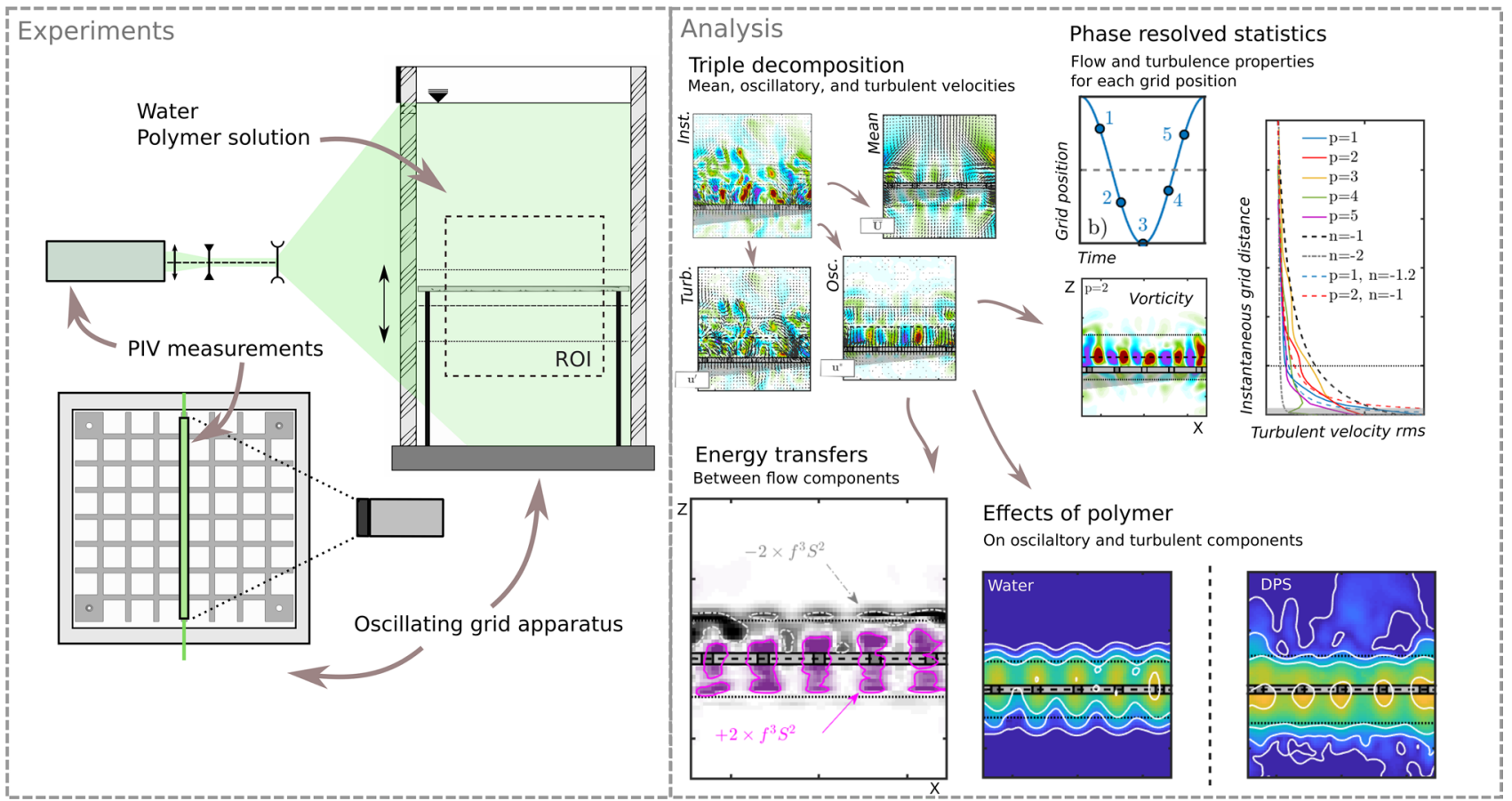

\section{Introduction}

A multitude of environmental phenomenon and industrial processes involve turbulent flows of complex liquid phases such as suspensions (Cuthbertson et al. 2018), multiphase media (Alméras et al. 2015), or liquids with complex rheology. The study of turbulence, already quite complex in itself, becomes even more challenging when it is done in such fluids. In particular, complex rheology liquids, known as non-Newtonian, are present in many industrial and environmental applications involving turbulent flows, for example in fermentation broths (Gabelle et al. 2013; Petř́iček et al. 2017). The complete understanding of complex real life situations requires fundamental description and characterization of turbulence properties in such fluids, and thus adequate experimental or numerical simplified cases of study. One of them is that of a rigid grid oscillating in a liquid initially at rest, called oscillating grid turbulence (OGT) (Hopfinger and Toly 1976; Thompson and Turner 1975).

Oscillating grid stirred tanks have been used for many purposes in research on turbulence. Among them is the study of interactions between turbulence and solid impermeable boundaries (McCorquodale and Munro 2017, 2018a), turbulence and gas-liquid mass transfer at a free surface (Brumley and Jirka 1987; Chiapponi et al. 2012; Herlina 2005; McKenna and McGillis 2004b), in stratified media (Hopfinger and Toly 1976; Thompson and Turner 1975; Verso et al. 2017; Xuequan and Hopfinger 1986), or study the behavior of bubbles, cells, fibres and aggregates suspended in a turbulent liquid phase (Mahamod et al. 2017; Nagami and Saito 2013; Rastello et al. 2017; San et al. 2017). Apart from fundamental turbulence studies, oscillating grids are used as flotation cells (Cuthbertson et al. 2018; Massey et al. 2012; Safari et al. 2017) in tanks with various shapes (tubular, prismatic, etc.) and with various number of grids.

Such devices are said to generate quasi-homogeneous and isotropic turbulence in horizontal planes (parallel to the grid, when it oscillates vertically), and to yield theoretically no mean flow, which is a major advantage compared to fixed grid setups in channels. The absence of a strong mean shear avoids some destruction of complex fluids' components (fibres, polymer chains, cells) that is sometimes observed in fixed grid turbulence (Vonlanthen and Monkewitz 2013). The fact that turbulent eddies are theoretically not advected by a strong mean flow makes them more easily observable and traceable by optical techniques such as particle image velocimetry (PIV) and planar laser-induced fluorescence (PLIF): seeding particles or tagged fluorescent molecules stay for longer times in the region of interest. 
However, the mechanisms of turbulence production by the oscillating grid itself have never been studied. It is commonly said that turbulence arises from the interaction of jets and wakes (Thompson and Turner 1975) behind grid holes and bars, but the exact interactions of these jets and wake, and the way they merge to generate homogeneous turbulence remained unobserved, even in water, until recently (McCorquodale and Munro 2018b).

Regarding oscillating grid turbulence in non-Newtonian liquids, only a few studies can be found in the literature (Liberzon et al. 2009; Wang et al. 2015, 2016), focused on purely elastic fluids, and none of them seems to tackle the effects of variable viscosity on either the mechanisms of turbulence generation, or the mean flow and turbulence properties mapping in the whole tank. This has only been described in a recent study by Lacassagne et al. (2019).

Studies of periodic and turbulent flows in non-Newtonian polymer solutions yet suggest that the presence of a minute amount of polymer in the liquid phase, conferring it either shear-thinning and/or viscoelastic properties, may significantly alter the energy distribution and exchanges between mean flow structures, oscillatory fluctuations and turbulence in the flow (Gabelle et al. 2013, 2017; Cocconi et al. 2017).

The aim of this paper is to study the influence of a shearthinning behavior on mean flow, oscillatory flows, and turbulence around an oscillating grid. This study is meant to bring additional information on both oscillating grid turbulence generation mechanisms, and on the fundamental effect of polymer induced shear-thinning property on oscillatory flows and turbulence.

The shear-thinning properties is given to an initially Newtonian fluid, water, by addition of a minute amount of polymer (xanthan gum, XG). Fluid velocity measurements are achieved using PIV in and around the region of the grid oscillations (grid swept zone). A triple decomposition methodology (Hussain and Reynolds 1970), already used in industrial impeller-stirred tank studies (Escudié and Liné 2003), is applied to reconstruct mean flows, oscillatory/periodic fluid motion, and turbulent velocity fluctuations. Oscillating grid flow in water is compared to a single polymer concentration case at the upper limit of the dilute regime.

The rest of present paper is organised in four sections. In the background section, the basis of oscillating grid turbulence in water is presented, the influence of polymer on turbulence in general and on grid turbulence (mostly for fixed grids) is described, and finally the triple decomposition method is introduced. In Sect. 3, the properties of polymer solutions used in this study are introduced and the oscillating grid setup is described. PIV measurements and image and data processing stages are also detailed. Section 4 first focuses on the decomposition of the velocity fields in mean, oscillatory and turbulent components, and on turbulent and periodic fluctuation properties. Energy transfers between flow components are then discussed, and further details on mean flow and turbulence generation are provided. Previous results are evidenced again by a viscosity estimation approach. In the conclusion, the mechanisms of flow generation by the oscillating grid in water are summarized, and the observed effects of polymer addition on the flow are listed.

\section{Background}

\subsection{Oscillating grid turbulence in water}

The principle of oscillating grid turbulence (OGT) is to produce turbulence in a fluid initially at rest by making a grid oscillate, usually vertically, at a frequency $f$ and with an amplitude or stroke $2 S$. It is commonly said that the jets and wakes behind the grid's holes and bars interact to generate turbulence (Herlina 2005; Hopfinger and Toly 1976; Thompson and Turner 1975; Voropayev and Fernando 1996), which then diffuses away from the grid. It is of great interest for turbulence study since it theoretically creates almost no mean flow and, therefore, allows to study the effects of turbulence alone, which is not the case for fixed grid systems. OGT is also supposed to yield a quasi-homogeneous and isotropic turbulence in horizontal planes when measured far enough from the grid's top position.

The first oscillating grid apparatus was designed by Rouse and Dodu (1955) to study turbulent diffusivity across a density stratification layer located above the grid and consisted in a square mesh grid in a cylindrical tank. Later, Bouvard and Dumas (1967) performed hot-fibre velocity measurements of grid generated turbulence in water, using a perforated plate as an oscillating grid. A full characterization of OGT in prismatic tanks came with the pioneer works of Thompson and Turner (1975) and Hopfinger and Toly (1976). Thompson and Turner (1975) studied several types of grids with different mesh sizes $M$ and different bar shapes, and showed that the best homogeneity and intensity of turbulence was achieved with square section bars and a solidity parameter $\Xi=\frac{d}{M}\left(2-\frac{d}{M}\right)$ lower than $0.4, d$ being the width of grid bars. Hopfinger and Toly (1976) added that the walls of the tank should be planes of symmetry for the grid, to minimize the recirculation effects in the tank. For the same reason, the grid's average position should be at least $2.5 \times M$ above the bottom of the tank (Xuequan and Hopfinger 1986). Horizontal homogeneity of turbulence is reached when the distance from the grid's mean position is greater than an empirically determined value $Z_{h}$ expressed in numbers of mesh parameter, with $2 M<Z_{h}<4 M$ depending on the study. Since the ultimate interest is to achieve turbulence homogeneity, many studies of OGT use this $Z_{h}$ parameter as a critical minimal distance at which the grid has to be placed 
from the item one wants to study the interaction with turbulence. Only recently, McCorquodale and Munro (2018b) evidenced the spatial homogenization of turbulence from the grid's swept zone to the $Z_{h}$ region. Once the far-grid, homogeneity region is reached, turbulence properties can be described by various evolution laws, first derived by Thompson and Turner (1975) and Hopfinger and Toly (1976) and later completed by other studies (Mcdougall 1979; Nokes 1988; Shy et al. 1997; Silva and Fernando 1994; Wan Mohtar 2016). These turbulence decay relationships are well described for water, but not in shear-thinning polymer cases. This yet will not be discussed in the present paper, as it is the subject of another work that has been published separately (Lacassagne et al. 2019). It is worth noting that OGT has almost never been simulated numerically. Only the recent work by Zhang et al. (2017) presents a Lattice Boltzmann simulation of OGT and numerically visualizes the interactions between jets and wakes behind the grid bars and holes leading to turbulence generation.

At first, the concept of OGT generating no mean flow but only isotropic and homogeneous turbulence was rather well believed. Yet with the development of PIV techniques allowing further investigations of the flow inside OGT tanks, it became clear that mean flow exist even when matching the requirements detailed above. McKenna and McGillis (2004b) studied the mean flows in an oscillating grid tank using PIV and showed that persistent mean flow cells always existed, with relative high kinetic energy level as compared to the turbulent kinetic energy. Moreover, these mean flows seem to be poorly reproducible and strongly dependent on initial conditions (Herlina 2005). It is, therefore, really hard to predict the mean flow that could occur in the oscillating tank during a specific measurement, and this is one of the main limitations of OGT systems.

A method for reducing mean flows in OGT is to use an inner box placed inside the stirred tank to separate the wall-induced vortices from the rest of the flow (Dickinson and Long 1983; Hopfinger and Toly 1976; McCorquodale and Munro 2018b). The complementary work previously referred to (Lacassagne et al. 2019) also suggests that the mean flow may be enhanced by the presence of polymer in the dilute entanglement regime. Investigations of flow properties in the grid region may allow to explain the origin of this mean flow both in water and in shear-thinning polymer solutions.

\subsection{Turbulence and grid turbulence in polymer solutions}

The influence of non-Newtonian behavior on grid turbulence has been mainly studied for fixed grid configuration and viscoelastic fluids (Barnard and Sellin 1969; van Doorn et al. 1999; Fabula 1966; Friehe and Schwarz 1970; Greated 1969;
McComb et al. 1977). Recently, Vonlanthen and Monkewitz (2013) used PIV measurements to look at turbulent spectra and scales in grid turbulence of dilute viscoelastic polymer solutions and evidence elastic modifications of turbulence. In their experiments, they found that both the shape of the energy spectrum and the elastic scale evolved with time, which they explained by the destruction of polymer chains by the strong shears in the vicinity of the grid. This shows one of the limits of fixed grid devices for the study of turbulence in polymer solutions: reaching high levels of turbulence requires high flow rate which causes important degradation of the polymer throughout the measurements. Moreover, turbulent velocity fluctuations are even smaller when compared to mean flow velocities than for the water case. One may thus prefer using OGT rather than fixed grid.

The first study of OGT in viscoelastic dilute polymer solutions (PEO) was made by Liberzon et al. (2009), who observed the propagation velocity of the boundary between turbulent and non-turbulent regions in the tank, at the first instants after the onset of the grid's oscillations. They found that the turbulent/non-turbulent interface moved globally faster in dilute polymer solution than in water. Wang et al. (2015) and Wang et al. (2016) later used a two oscillating grid system to study the viscoelastic effects of surfactants and dilute polymers on coherent structures. Using proper orthogonal decomposition (POD), they show that the addition of polymer tends to decrease the small-scale effects of turbulence, and that this decrease can not only be attributed to the overall viscosity increase, since it is not associated with a decrease of the turbulent kinetic energy (TKE). Hence, the non-Newtonian property of the flow seems to strongly modify the spectrum behavior of the turbulent structures. They show promising results for the use of this method to analyze OGT flow properties in complex fluids such as DPS.

\subsection{Triple decomposition of turbulence in periodic flows}

In some cases, a flow may display a periodic behavior due to external forcing or waves. Blade motion in stirred tanks (Escudié and Liné 2003), periodic vortex shedding at rigid boundaries in free surface flows (Mignot et al. 2016), or pulsating flow in blood vessels induced by periodic heartbeats (Holdsworth et al. 1999) are good examples of flows in which an oscillatory/organised/periodic effect is present. Oscillating grid turbulence used in this work is another one. In such flows, the time average of the oscillatory component of the flow is nil. Hence, when using classical Reynolds decomposition to describe them, periodic fluctuations are included considered together with while they are not strictly speaking turbulent fluctuations. 
To be able to distinguish turbulence from organised motion, Hussain and Reynolds (1970) proposed a triple decomposition of the velocity field, that will be called triple decomposition hereinafter. The total instantaneous velocity field is this time written as the sum of the average velocity $\bar{U}$, the periodic fluctuation $u^{*}$ and the turbulent fluctuation $u^{\prime}$ :

$U(M, t)=\bar{U}(M, t)+u^{*}(M, t)+u^{\prime}(M, t)$.

Pressure decomposes the same way, with $\overline{u^{*}}=0, \overline{u^{\prime}}=0$, $\overline{p^{*}}=0, \overline{p^{\prime}}=0, \overline{u^{*} u^{\prime}}=0$ and $\overline{p^{*} p^{\prime}}=0$. Turbulent, mean and oscillatory kinetic energies can be defined, respectively, as $k^{\prime}=\frac{1}{2} \overline{u_{i}^{\prime} u_{i}^{\prime}}=, K=\frac{1}{2} \bar{U}_{i} \bar{U}_{i}$ and $k^{*}=\frac{1}{2} \overline{u_{i}^{*} u_{i}^{*}}$. Hussain and Reynolds (1970) derived the following governing equations for, respectively, $k^{\prime}, k^{*}$ and $K$ :

$$
\begin{aligned}
& \underbrace{\frac{\partial k^{\prime}}{\partial t}}_{(1)}+\underbrace{\bar{U}_{j} \frac{\partial k^{\prime}}{\partial x_{j}}}_{(2)}=-\underbrace{\frac{1}{\rho} \frac{\partial}{\partial x_{j}}\left[\overline{u_{j}^{\prime}\left(p^{\prime}+\frac{1}{2} \rho u_{i}^{\prime} u_{i}^{\prime}\right)}\right]}_{(3)} \\
& +\underbrace{\left(-\overline{u_{i}^{\prime} u_{j}^{\prime}}\right) \frac{\partial \bar{U}_{i}}{\partial x_{j}}}_{(4)}+\underbrace{\overline{\left(-\left\langle u_{i}^{\prime} u_{j}^{\prime}\right\rangle_{\phi} \frac{\partial u_{i}^{*}}{\partial x_{j}}\right)}}_{(5)} \\
& +\underbrace{\overline{u_{j}^{*} \frac{\partial}{\partial x_{j}}\left\langle\frac{1}{2} u_{i}^{\prime} u_{j}^{\prime}\right\rangle_{\phi}}}_{(6)}+\underbrace{\left.v \frac{\partial}{\partial x_{j}} \overline{u_{i}^{*}\left(\frac{\partial u_{i}^{\prime}}{\partial x_{j}}+\frac{\partial u_{j}^{\prime}}{\partial x_{i}}\right)}\right]}_{(7)} \\
& -\underbrace{\frac{v}{2\left(\frac{\partial u_{i}^{\prime}}{\partial x_{j}}+\frac{\partial u_{j}^{\prime}}{\partial x_{i}}\right)^{2}}}_{(8)}
\end{aligned}
$$

where (1) is the accumulation term, (2) is advection by the mean flow,(3) (6) and (7) are diffusion terms, term (8) dissipation, and terms (4) and (5) are exchanges with mean and oscillatory components. $\langle a\rangle_{\phi}$ is the phase-averaged quantity of $a$ (see Sect. 3.4).

$$
\begin{aligned}
& \underbrace{\frac{\partial k^{*}}{\partial t}}_{(1)}+\underbrace{\bar{U}_{j} \frac{\partial k^{*}}{\partial x_{j}}}_{(2)}=-\underbrace{\frac{1}{\rho} \frac{\partial}{\partial x_{j}}\left[u_{j}^{*}\left(p^{*}+\frac{1}{2} \rho k^{*}\right)\right]}_{(3)} \\
& +\underbrace{\left(-\overline{u_{i}^{*} u_{j}^{*}}\right) \frac{\partial \bar{U}_{i}}{\partial x_{j}}}_{(4)}-\underbrace{\overline{\left(-\left\langle u_{i}^{\prime} u_{j}^{\prime}\right\rangle_{\phi} \frac{\partial u_{i}^{*}}{\partial x_{j}}\right)}}_{(5)} \\
& +\underbrace{\left.v \frac{\partial}{\partial x_{j}} \overline{u_{i}^{*}\left(\frac{\partial u_{i}^{*}}{\partial x_{j}}+\frac{\partial u_{j}^{*}}{\partial x_{i}}\right)}\right]}_{(6)}-\underbrace{\frac{v}{2\left(\frac{\partial u_{i}^{*}}{\partial x_{j}}+\frac{\partial u_{j}^{*}}{\partial x_{i}}\right)^{2}}}_{(7)},
\end{aligned}
$$

where term (1) is the accumulation term, term (2) is advection by the mean flow, terms (3) and (6) are diffusion, term (7) is dissipation, and terms (4) and (5) are exchanges with mean and turbulent components.

$$
\begin{aligned}
& \underbrace{\frac{\partial K}{\partial t}}_{(1)}+\underbrace{\bar{U}_{j} \frac{\partial K}{\partial x_{j}}}_{(2)}=-\underbrace{\frac{1}{\rho} \frac{\partial \bar{U}_{i} \bar{P}}{\partial x_{i}}}_{(3)} \\
& -\underbrace{\left(-\overline{u_{i}^{\prime} u_{j}^{\prime}}-\overline{u_{i}^{*} u_{j}^{*}}\right) \frac{\partial \bar{U}_{i}}{\partial x_{j}}}_{(4)}-\underbrace{\frac{\partial}{\partial x_{j}}\left[\bar{U}_{i}\left(-\overline{u_{i}^{\prime} u_{j}^{\prime}}-\overline{u_{i}^{*} u_{j}^{*}}\right)\right]}_{(5)} \\
& +\underbrace{v \frac{\partial}{\partial x_{j}}\left[\bar{U}_{i}\left(\frac{\partial \bar{U}_{i}}{\partial x_{j}}+\frac{\partial \bar{U}_{j}}{\partial x_{i}}\right)\right]}_{(6)} \\
& -\underbrace{\frac{\nu}{2}\left(\frac{\partial \bar{U}_{i}}{\partial x_{j}}+\frac{\partial \bar{U}_{j}}{\partial x_{i}}\right)^{2}}_{(7)},
\end{aligned}
$$

where term (1) is the accumulation term, term (2) is advection by the mean flow, term (3) is the pressure velocity correlation, terms (5) and (6) are diffusion terms, term (7) is dissipation, and term (4) is the transfer to other components' kinetic energy. From Eqs. (2), (3) and (4), it is possible to extract the exchange terms (respectively numbered (5), (4) and (5), and (4)) and identify the expression of the kinetic energy transfer between each components of the velocity field (Escudié and Liné 2003):

Mean to turbulent energy transfer:

$\mathscr{T}_{\mathrm{mt}}=-\overline{u_{i}^{\prime} u_{j}^{\prime}} \frac{\partial \bar{U}_{i}}{\partial x_{j}}$.

Mean to oscillatory energy transfer:

$\mathscr{T}_{\mathrm{mo}}=-\bar{u}_{i}^{*} u_{j}^{*} \frac{\partial \bar{U}_{i}}{\partial x_{j}}$.

Oscillatory to turbulent energy transfer:

$\mathscr{T}_{\mathrm{ot}}=\overline{\left(-\left\langle u_{i}^{\prime} u_{j}^{\prime}\right\rangle_{\phi} \frac{\partial u_{i}^{*}}{\partial x_{j}}\right)}$.

Each of these transfer terms is a product of a gradient of mean or oscillatory motion and a stress term of organized and turbulent motion. The direct transfer from mean flow to turbulent motion $\mathscr{T}_{\mathrm{mt}}$ is equivalent to the turbulence production by mean flow in Reynolds classical decomposition. However, in the present case, the mean flow also transfers part of its energy to the periodic one $\left(\mathscr{T}_{\text {mo }}\right)$ and part of the 

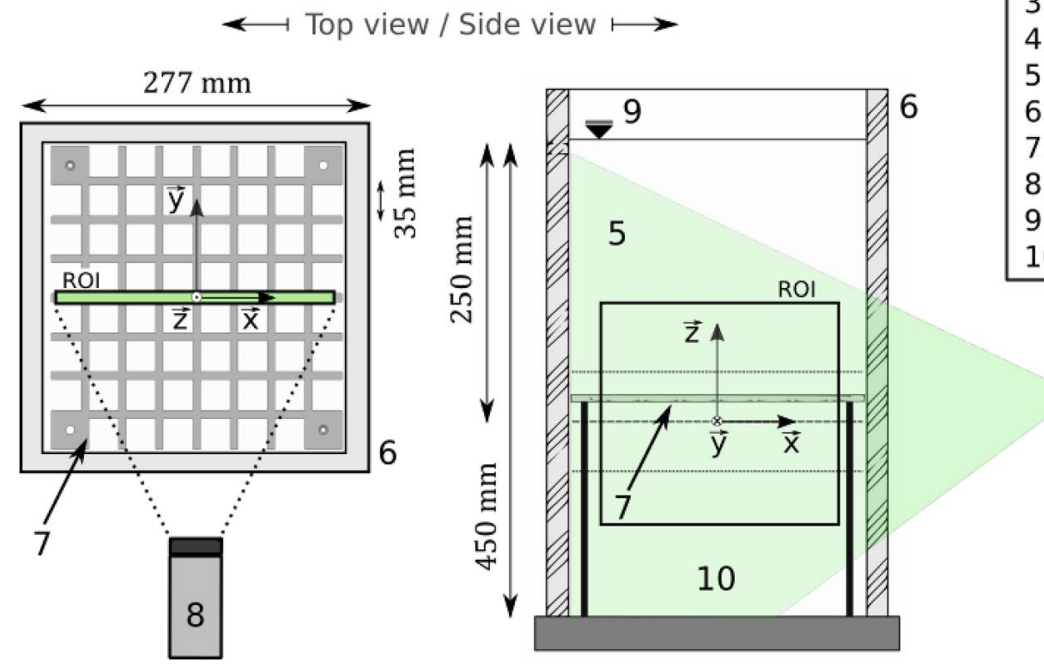

1. Nd:YAG Laser

2. Converging spherical lens

3. Diverging spherical lens

4. Cylindrical diverging lens

5. Laser sheet

6. Tank

7. Oscillating grid

8. CCD cameras + lens

9. Free surface

10. Water / DPS

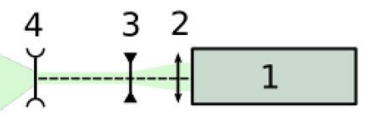

Fig. 1 Sketch of the oscillating grid and PIV setup

turbulent kinetic energy production comes from the organized motion $\left(\mathscr{T}_{\text {ot }}\right)$. To fully understand turbulence in oscillatory flows, the knowledge of these transfer terms is required. It is worth noting that all of the above equations reduce to the ones obtained using the classical Reynolds decomposition when $u^{*}<<u^{\prime}$, i.e., in case of negligible oscillatory flow influence.

\section{Materials and methods}

\subsection{Polymer solutions}

Shear-thinning properties are conferred to the liquid by addition of a minute amount of a polymer, xanthan gum (XG), to distilled water. Here $\mathrm{XG}$ produced by Kelco under the commercial name Keltrol CG-T is used. Its average molar mass is $M_{w}=3.4 \times 10^{6} \mathrm{~g} \mathrm{~mol}^{-1}$ and its polydispersity equal to 1.12 (Rodd et al. 2000). XG is chosen for its high resistance to strong shears and extreme temperature and $\mathrm{pH}$ conditions (Garcia-Ochoa et al. 2000). Such features are useful when using it nearby a rigid oscillating grid. XG yields optically clear non-Newtonian solutions once dissolved in aqueous media. This allows the use of optical methods for liquid phase velocity measurements. This polymer is commonly utilized as a flow additive for non-Newtonian fluid mechanics experiments, but also in the food, process, and oil and gas industries (Katzbauer 1998). Its rheology and its properties have been widely studied in the literature (Cuvelier and Launay 1986; Wyatt and Liberatore 2009; Wyatt et al. 2011). Depending on the polymer concentration $C_{X G}$, one may distinguish between three entanglement regimes: dilute, semi-dilute, and concentrated (Cuvelier and Launay 1986; Wyatt and Liberatore 2009). In the last two cases, the flow behavior is defined by fluid-polymer but most importantly polymer-polymer mechanical or electrical interactions. In this work the focus is made on the dilute regime, for which polymer molecules only interact with the flow and not between each other. It concerns the $C_{X G} \leq 100 \mathrm{ppm}$ concentration range (Wyatt and Liberatore 2009). Here the working concentration is $C_{X G}=100 \mathrm{ppm}$ (Fig. 1). This last concentration is chosen so that the solution is still in the dilute regime for entanglement, with the strongest possible concentration effects within that regime. One then talks about a dilute polymer solution (DPS). The shear-thinning behavior of aqueous XG DPS can be modelled by a Carreau-Yasuda (CY) equation

$\frac{\mu-\mu_{\infty}}{\mu_{0}-\mu_{\infty}}=\left(1+\left(t_{\mathrm{CY}} \dot{\gamma}\right)^{a}\right)^{\frac{n-1}{a}}$.

With $\mu_{0}=32.8 \mathrm{mPa}$ s and $\mu_{\text {inf }}=1.1 \mathrm{mPa}$ s the zero shear rate and infinite shear rate Newtonian plateau, $t_{\mathrm{CY}}=1.58 \mathrm{~s}$ a characteristic timescale of the polymer, $(n-1)=-0.5$ the shear-thinning power law exponent, and $a=2$ a parameter for the transition between power law and Newtonian behaviors (all values given for $C_{X G}=100 \mathrm{ppm}$ ). The evolution of viscosity with the nominal shear rate is shown on Fig. 2. Vertical lines indicates estimate of (1) the typical shear rates caused by the grid (full line, based on its frequency), and (2) the maximum shear rates caused by mean, oscillatory and turbulent motions (based on data shown in Fig. 7). Viscoelasticity is checked to be negligible for such 


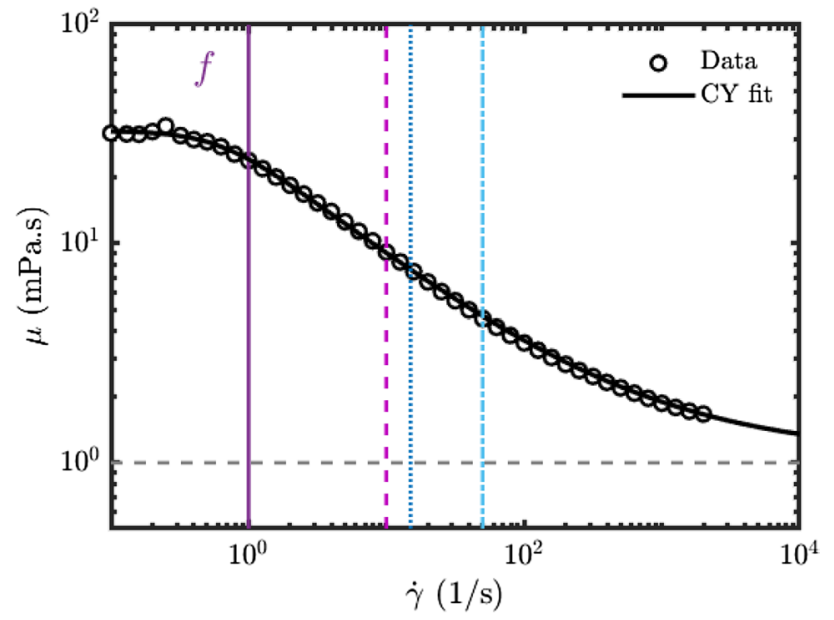

Fig. 2 Viscosity data points (circles) and Carreau-Yasuda fitting (line) for the $100 \mathrm{ppm}$ XG solution. The horizontal dashed line denotes the viscosity of water. The vertical full line is the nominal shear rate corresponding to the grid frequency. Vertical dashed, dotted, and mixed lines show, respectively, the order of magnitude of the maximum mean, oscillatory, and turbulent velocity gradients. They thus indicate the range of viscosity variations induced by all three types of flow

a XG concentration. Steady shear rheological measurements performed before and after experiments allow to check that polymer chains are not destroyed by grid oscillations, or at least that destruction does not affect the shear-thinning property (see Lacassagne (2018), Fig. 3.10).

\subsection{Oscillating grid setup}

Turbulence is generated in a transparent prismatic tank of $L^{2}$ inner cross section with $L=277 \mathrm{~mm}$. The fluid height is set at $H=450 \mathrm{~mm}$ and the distance between the surface and the average grid position is $250 \mathrm{~mm}$. The vertical axis, oriented upwards, is noted $Z$, and $X$ and $Y$ are the axis defined by the grid bar (see Fig. 1). The origin of the reference frame is placed at the grid average position $(Z=0)$ at the crossing between the two central bars. In this study, only polymer concentration is varied and all oscillation parameters are kept constant. The grid bars are $d=7 \mathrm{~mm}$ thick and the mesh parameter is $M=35 \mathrm{~mm}$. The frequency is fixed at $1 \mathrm{~Hz}$ and the amplitude at $2 S=5 \mathrm{~cm}$, This allows to define a grid-based Reynolds number using the definition of Janzen et al. (2010), but based on the zero shear rate viscosity $\mu_{0}$ :

$R e_{g}=\frac{\rho f(2 S)^{2}}{\mu_{0}}$

The density $\rho$ of the fluid is assumed equal to that of water, because of the very small mass of polymer added. Here one gets $R e_{g}=2500$ for water and $R e_{g}=76$ for the polymer case. To quantify the ratio between the polymer relaxation timescale and the grid forcing timescale the grid-based Deborah number De is defined as

$D e=\frac{t_{\mathrm{CY}}}{T}=t_{\mathrm{CY}} f$,

with $\mathrm{T}$ the period of oscillations. Here $D e=1.58$.

\subsection{PIV measurements}

Liquid phase velocity measurements in the tank are achieved by particle image velocimetry (PIV). The experimental setup is sketched in Fig. 1. The region of interest (ROI) is a vertical rectangle, in a plane normal to the central bar of the oscillating grid. Its width is close to that of the tank, and it is vertically centred around the grid swept zone. The cameras used is a double frame LaVision SCMOS sensors of 2560 by 2160 pixels, equipped with a $50 \mathrm{~mm}$ focal macro lens. A pulsed Quantel Nd:YAG laser emitting at $\lambda=532 \mathrm{~nm}$ is used to illuminate $50 \mu \mathrm{m}$ diameter polyamide particles. Double-frame PIV at an acquisition frequency $f_{\text {acq }}$ of $10 \mathrm{~Hz}$ is performed. The time interval between laser pulses is $\Delta t=4 \mathrm{~ms}$. Vector fields are computed with DaVis 8 software using a multipass processing: a first pass with 48 by 48 pixels and 2 following passes with 24 by 24 pixels round Gaussian weighted interrogation windows, at a maximum of $50 \%$ overlapping. Spurious vectors are removed from PIV fields by applying a threshold of 1.2 on the peak ratio, and replaced using median filtering.

The final spatial resolution achieved is $3.4 \mathrm{~mm}$. The smallest Kolmogorov length scale and Taylor micro-scales of turbulence are supposed to be found in the water case, for which the viscosity is always the lowest. The Kolmogorov and Taylor length scales are evaluated to be, respectively, of about $0.47 \mathrm{~mm}$ and $3.17 \mathrm{~mm}$ for water. For $100 \mathrm{ppm}$ XG solutions, using a constant viscosity equal to $\mu_{\infty}$, they increase to $0.23 \mathrm{~mm}$ and $3.25 \mathrm{~mm}$, and up to $6.45 \mathrm{~mm}$ and $18.17 \mathrm{~mm}$ when using $\mu_{0}$ as the scale viscosity. The $3.4 \mathrm{~mm}$ spatial resolution is thus quite coarse and would unfortunately not allow to evidence energy variations at large wave numbers characteristic of viscoelastic turbulence. We shall thus discuss only the inertial and large scale effects.

Measurements performed here are planar (2D PIV) and give access to only two components of the velocity field. As a consequence, it is not possible to compute all the terms of the governing equations introduced in Sect. 2.3, but only a part of the terms involving the components and their gradients along $X$ ( $i$ and/or $j$ equal to $x$ ) and $Z$ ( $i$ and/or $j$ equal to $z$ ). In what follows, analysis of the velocity fields is carried out as if the flow was two dimensional, assuming that velocities and gradients along the $y$ direction are nil. This is a strong and reductive hypothesis. To perform a complete 

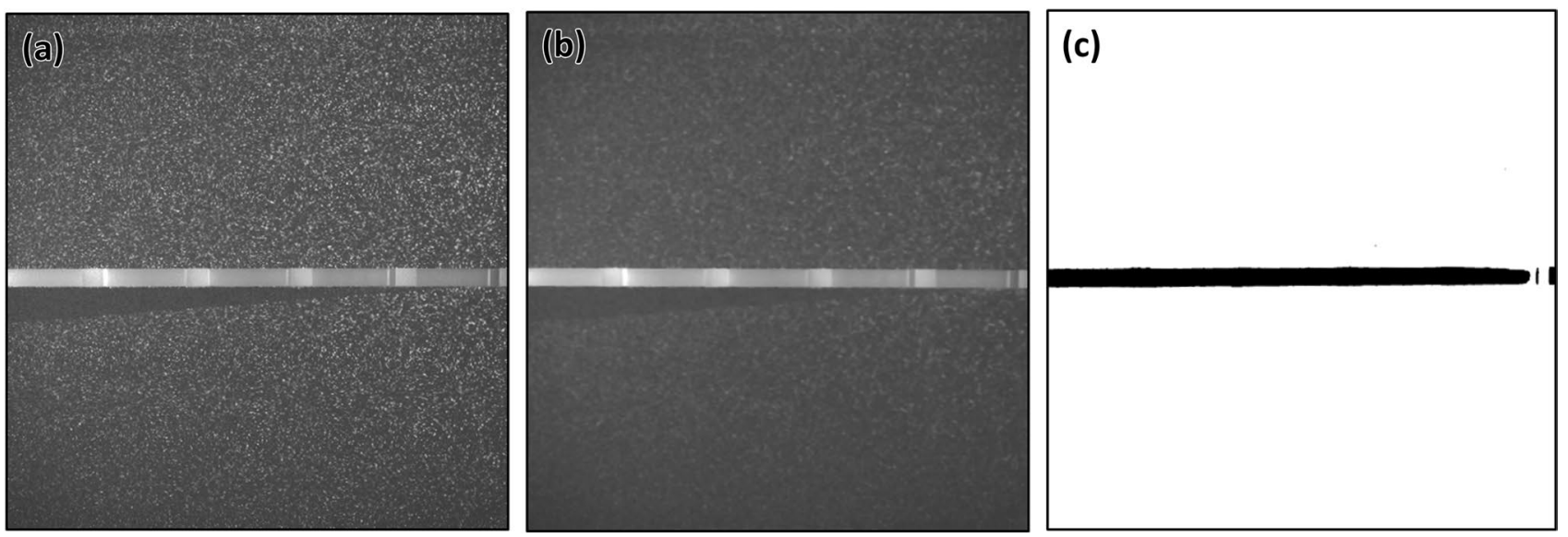

Fig. 3 Grid masking procedure. a Recorded image showing both the grid and PIV particles, b particles smoothed out by applying an horizontal median filter, $\mathbf{c}$ mask obtained using an arbitrary intensity threshold. The laser sheet comes from the right, as sketched in Fig. 1

analysis of the production, dissipation, and transfer terms of turbulence of the governing equations in the whole volume of interest, full three-dimensional three-component velocity data would be required.

\subsection{Grid detection and phase-resolved statistics}

\subsubsection{Grid masking and tracking}

As measurements are focused around the swept zone, the oscillating grid is visible on the recorded images (Fig. 3a), which is an asset for grid tracking but problematic for the velocity field calculation. The grid has, therefore, to be first removed from images for velocity calculation and then isolated for the grid tracking step. Grid masking is achieved using DaVis software from LaVision. At first, an horizontal median filter is applied on every recorded image (Fig. 3b). These images are then binarized using an arbitrary threshold based on the image gray level values such that the pixel corresponding to the grid are set to zero and the rest of the image to one (Fig. 3c). Each mask image is then applied to the corresponding image when calculating the velocity field. One effect that cannot be easily corrected with this procedure is the shaded area visible on the left part of Fig. 3a under the grid. Here the PIV laser sheet was directed so that it always met the grid from the top, and the shaded region was always under the grid. In doing so, the region above the grid is always well lighted, and measurements can always be considered reliable there. The shaded particle region is represented by a gray zone on each PIV-deduced fields at specific grid positions in the following figures, to recall that this region is prone to errors.

Grid tracking is performed by a Matlab custom script. It also starts by an horizontal median filtering step to smooth particles off the image. Gray level intensity is then horizontally averaged on the whole image width, and the peaks of width-averaged intensity gradients are used to detect grid boundaries (Fig. 4). Finally, the grid position is defined at equal distance from the two peaks. Once the grid tracking step is made, every pair of PIV frames and the corresponding vector field can be associated with a grid position. The grid direction (going up or going down) for each instantaneous fields can be found using the difference in detected grid position on frames 1 and 2 .

\subsubsection{Conditional averaging and triple decomposition}

As mentioned in Sect. 2.3, a triple decomposition of the velocity field into mean, oscillatory, and turbulent

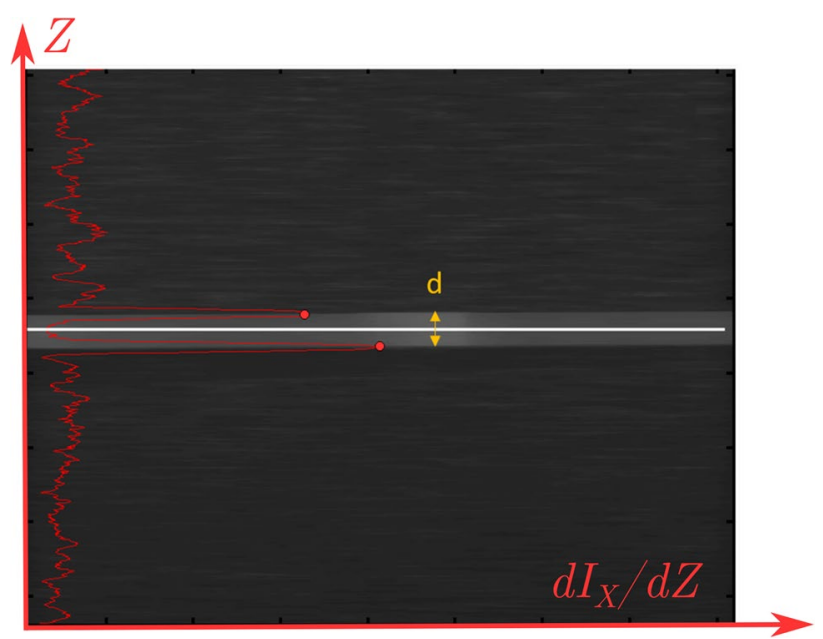

Fig. 4 Grid tracking procedure: the red curve is the vertical gradient of horizontally averaged intensity $I_{X}$. The two red dots correspond to the maximum gradient peak detected. It is checked that the distance between the two peaks is equal to the grid thickness d (orange). The white line shows the detected grid position 
components is a useful tool to study the dynamics of flows which exhibit a periodic pulsation or sustained by a periodic forcing. Escudié and Liné (2003) detailed a method to extract mean, oscillatory and turbulent component of the flow from statistical analysis of non-time-resolved 2D-PIV measurements synchronized on blade motion. The main idea was to perform different sets of PIV measurements corresponding each to a different but known position of the blade (or here the grid), the synchronization between blade/grid position and measurement being enforced by an outside detection system. Here the method used to achieve phaseresolved measurements is not based on an external trigger but rather on image processing of a collection of randomly sampled instantaneous fields. The main principle of statistical estimation of the triple decomposition component still stays the same, and is detailed in what follows.

Sampling of the grid stroke A conditional averaging of velocity fields is performed based on the grid position previously detected. To that end, the total measured amplitude is sampled into $N_{\mathrm{p}}$ parts of constant size $\Delta S$ such that $2 S=N_{\mathrm{p}} \times \Delta S$. Based on its measured grid position, each instantaneous field is then associated to the slice it lies into, and is thus affected to a closest matching sample grid position. Phase averaging is performed by averaging all the velocity fields stored at a given sampling grid position, (i.e., in a given slice). Ultimately, the typical period of oscillation is described using $2 \times N_{\mathrm{p}}-1$ point in time ( $N_{\mathrm{p}}$ for the grid going up, same for the grid going down, minus the starting point). $N_{\mathrm{p}}$ is chosen as the best compromise between high period sampling and convergence of each position's velocity statistics. Indeed, if $N_{\mathrm{p}}$ is too large, each slice may not include enough instantaneous field to achieve statistical convergence (see the next paragraph). On the other hand, if $N_{\mathrm{p}}$ is too low, one may not be able to describe accurately oscillatory velocity fields around the grid, and results can not be considered phase-resolved. The results presented hereinafter are for $N_{\mathrm{p}}=12$. This induces every slice to always store at least 250 instantaneous data fields, which has proven to be sufficient for phase-averaged velocity fields calculation (see Fig. 5 and following paragraphs).

Flow statistics Knowing the phase-averaged velocity fields, it is then possible to perform a triple decomposition of the velocity field as proposed by Hussain and Reynolds (1970) and explained in Sect. 2.3. It is worth noting that triple decomposition has never been applied to the study of oscillating grid turbulence despite its obvious oscillatory behavior.

Here for a velocity component $i$, the mean velocity field is taken as the ensemble average of all velocity fields:

$\bar{U}_{i}=\frac{1}{N} \sum_{p=1}^{N} U_{i}^{p}$,

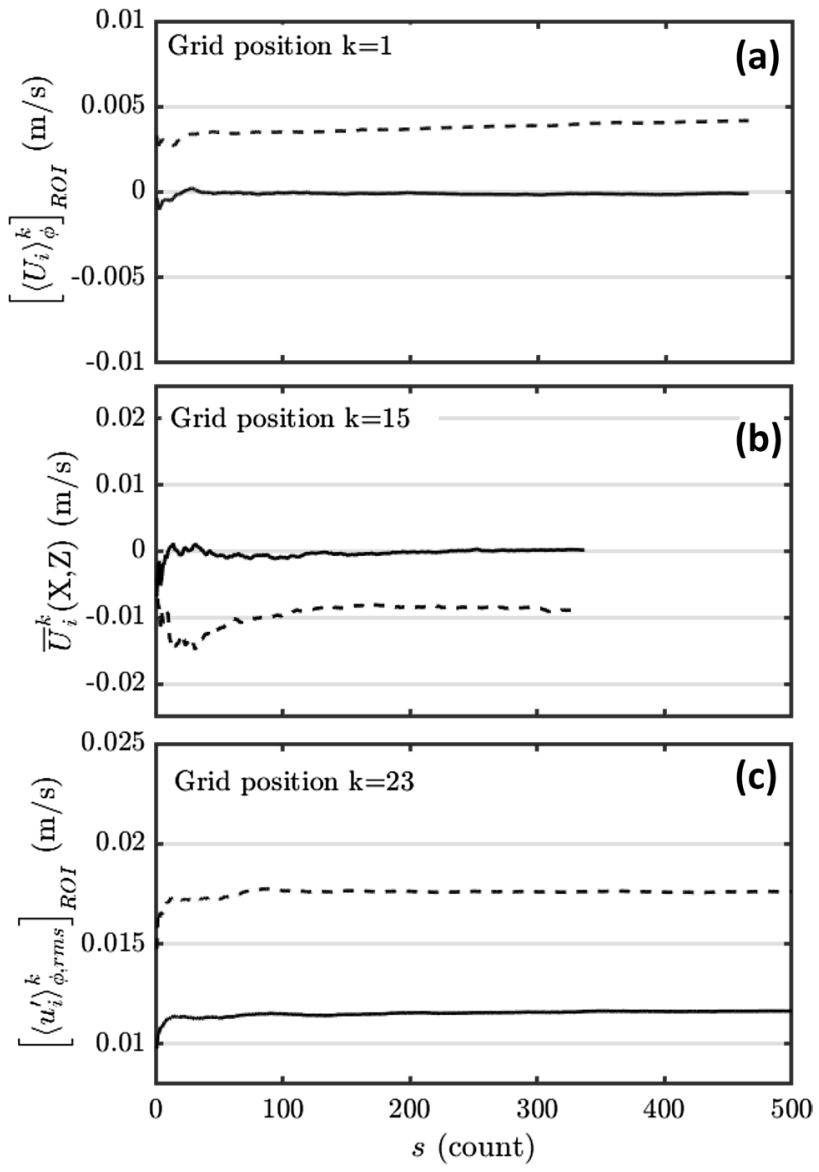

Fig. 5 Example of graph of convergence of statistical quantities (water). $s$ is the size of sample used for statistical analysis. a Convergence of the phase-averaged velocity values, spatially averaged over the whole ROI. b Statistical convergence of the same quantity at a given probing location $(X, Z)$ of the ROI (here $X=0, Z / S=2)$. c Convergence of the phase rms velocities, averaged over the ROI. Full lines are for horizontal velocity components and dashed lines for vertical velocity components. Examples are provided for three grid positions ( $k=1$ for $\mathbf{a}, k=15$ for $\mathbf{b}$ and $k=23$ for $\mathbf{c}$ ) but similar results are achieved for all $2 N p-1=23$ possible grid sampling positions with all three $(\mathbf{a}-\mathbf{c})$ indicators and for both fluids. []$_{R O I}$ denotes a spatial average over the full ROI

where $N$ is the total number of instantaneous velocity fields. Phase-averaged velocity for a given grid position $\mathrm{k}$ is defined as

$\left\langle U_{i}\right\rangle_{\phi}^{k}=\frac{1}{N_{k}} \sum_{p=1}^{N_{k}} U_{i}^{p}$,

With $N_{k}$ the number of instantaneous fields $U^{p}$ at grid position $k\left(U^{p=k}\right)$ in the phase.

The oscillatory component at this same grid position is the phase-averaged velocity from which the mean component is removed: 
$u_{i}^{* k}=\frac{1}{N_{k}} \sum_{p=1}^{N_{k}}\left(U_{i}^{p}-\bar{U}_{i}\right)$

The turbulent fluctuation accounting for the grid position $\mathrm{k}$ is thus:

$u_{i}^{\prime k}=U_{i}-\bar{U}_{i}-u_{i}^{* k}$.

The phase root mean square (rms) velocity can then be defined as the root mean square of turbulent fluctuations at position $\mathrm{k}$ :

$\left\langle u_{i}^{\prime}\right\rangle_{\phi, \mathrm{rms}}^{k}=\sqrt{\frac{1}{N_{k}} \sum_{p=1}^{N_{k}}\left(u_{i}^{\prime p}\right)^{2}}$.

It can be used to estimate the intensity of turbulence at position k, and will be later referred to as "phased rms" Finally, the non-phase-averaged rms of oscillatory and turbulent velocity fluctuations are defined, respectively, as

$\left\langle u_{i}^{*}\right\rangle_{\mathrm{rms}}=\sqrt{\frac{1}{N_{\mathrm{p}}} \sum_{p=1}^{N_{\mathrm{p}}}\left(u_{i}^{* p}\right)^{2}}$

and

$\left\langle u_{i}^{\prime}\right\rangle_{\mathrm{rms}}=\sqrt{\frac{1}{N} \sum_{p=1}^{N}\left(u_{i}^{\prime p}\right)^{2}}$.

They quantify for a given Eulerian probing point the intensity of respectively oscillatory and turbulent fluctuations at a fixed location in the flow.

Statistical convergence and data weight/size For all quantities, it has to be checked that statistical convergence is reached. The number of independent instantaneous fields used for average or rms computation on the whole set of data $(N)$ or at the $k$ position $\left(N_{k}\right)$ should be large enough for the computed quantity to be independent of it. This is verified by computing the sliding statistics using larger and larger $\mathrm{N}$ until the chosen indicator for statistical convergence reaches a constant value. ${ }^{1}$ For non-time-resolved PIV measurements of turbulent flows, it is commonly said that a few hundreds of independent fields are sufficient to reach convergence of averaged quantities, and about 1000 is required for secondorder statistical quantities (rms, for example). This criterion is yet arbitrary, so statistical convergence has been checked for our measurements. Our phase-resolved measurement provide uncorrelated fields for phase rms computation since

\footnotetext{
${ }^{1}$ A more rigorous approach could be lead via an ensemble probability estimation method (Simoëns 1992), in which the amount of independent field needed to achieve statistical convergence is predetermined by the analysis of the expected shape of concentration fluctuations PDF.
}

two fields recorded at the same grid position are separated by a minimum amount of time of $1 \mathrm{~s}$ corresponding to at least one grid period. To perform phase-averaged statistical analysis, a minimum of a few hundreds of images is needed for each grid sample position. The typical total number of images recorded here to fulfil this requirement is thus around 10,000 , with a number of images per grid position $N_{k}$ laying between 250 and 500 . Here $N_{k}$ is a consequence of the true grid frequency, the acquisition frequency of the PIV system, the lag between these two frequencies and the accuracy of the grid detection procedure.

Statistical convergence is checked at all grid sample positions. To that end, the average and rms values are plotted as a function of the sample size $N$ or $N_{k}$ for all velocity components (along $X$ and $Z$ ), at several random locations in the ROI or spatially averaged over the whole ROI. An example of a convergence graph is shown in Fig. 5. Globally, all indicators are found to converge in all ROIs and planes.

\section{Results and discussion}

\subsection{Flow visualization}

\subsubsection{Instantaneous velocity fields and flow decomposition}

An example of instantaneous velocity field for each fluid is pictured in Fig. 6 for a bottom grid position. In this figure as for all following fields, the $Z$ dimension is scaled by the stroke $S$, and the $X$ dimension by the semi width of the $\operatorname{tank} L / 2$. In both cases, the wake of the grid is evidenced by a series of counter rotating vortices behind each grid bars. DPS visually exhibits stronger but larger vortices than water. Using the grid detection procedure presented in Sect. 3.4.1, each instantaneous field is associated to a specific grid position. The mean velocity field is computed by averaging all instantaneous velocity fields, and phase-averaged velocity fields by averaging all instantaneous velocity fields at the same grid position, as explained in Sect. 3.4.2. The oscillatory component is deduced by removing the mean velocity field from phase-averaged velocity field at each grid position, and fluctuating velocity fields are derived by subtraction of mean and oscillatory fields to each instantaneous field.

Figure 7 gathers, for the two studied fluids, their mean velocity field, a phase-averaged and oscillatory velocity field at a given grid position (bottom position), and the fluctuating velocity field corresponding to instantaneous fields that are shown in Fig. 6. The background color represents the vorticity of each velocity field, respectively $\bar{\Omega},\langle\Omega\rangle_{\phi}, \Omega^{*}$ and $\Omega^{\prime}$ for the average, phase-averaged, oscillatory and fluctuating vorticity. The averaged fields show two side vortex above the grid swept zone. They 


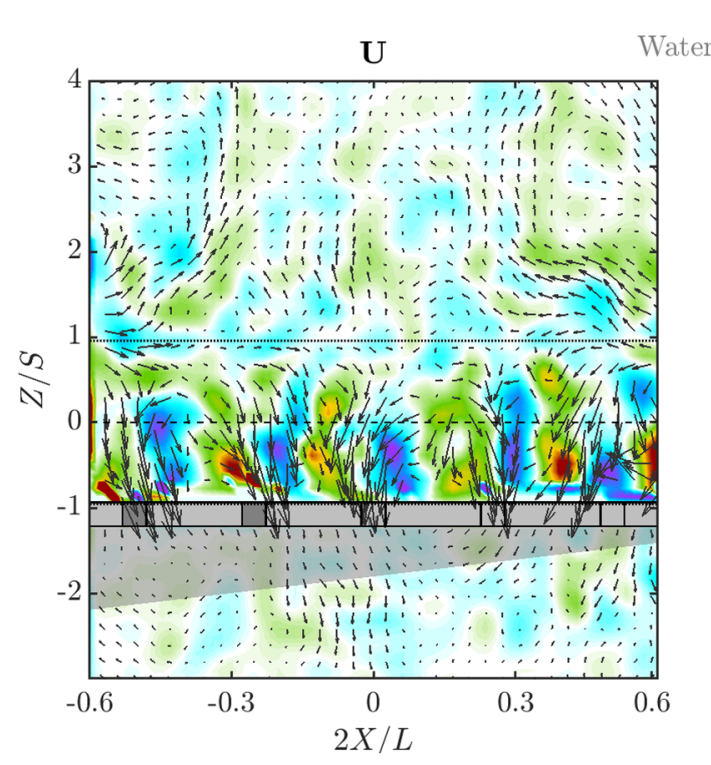

Fig. 6 Example of close grid instantaneous velocity and vorticity fields in water (left) and dilute polymer solution (DPS) (right). The color bar represents the out-of-plane vorticity of $U$. The gray area under the grid represents the area of the ROI for which the laser sheet

seem stronger in DPS than in water, and accompanied, in DPS only, by an enhanced up-going motion above the swept zone centred around $X=0$. This specific mean flow enhancement is described in Lacassagne et al. (2019). Phase average velocity field exhibit more clearly the grid bar wakes, together with the side vortices. The oscillatory component mostly displays the wakes since it is the result of the subtractions between phase-averaged measurement and mean flow. A new interesting difference between water and DPS appears when comparing the oscillatory components at the low grid position: DPS fields display two symmetric pairs of vorticity patches at the edges of the ROI above the grid swept zone, which are not visible for water. Finally, the fluctuating velocity fields allow to see the turbulent structures produced behind the grid. Eddies are created close to the grid and interact so that turbulence diffuses towards the bulk.

\subsubsection{Typical period of oscillations}

The typical period of oscillations can be described by considering the phase-averaged velocity field $\langle U\rangle_{\phi}$ (and its associated vorticity), to which we subtract the mean flow $\bar{U}$ (and its vorticity). Figure 8 displays a typical period of oscillations for the resulting oscillatory flow illustrated by the oscillatory component of the vorticity field. Five successive grid positions $k=p$ are sketched (a snapshot has already been presented in Fig. 7 for the lower grid position).

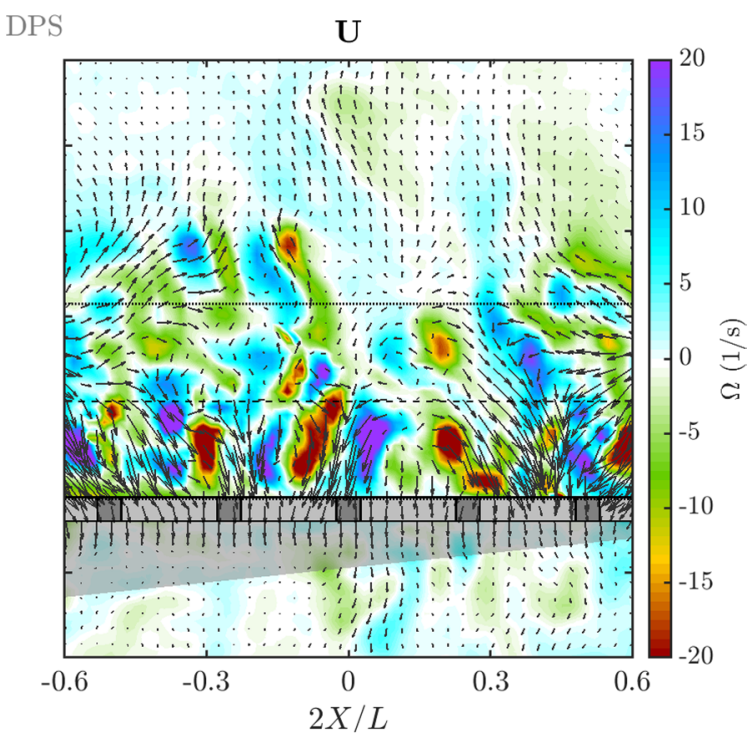

was shaded by the grid and were measurements could be inaccurate. For figure's clarity, only one every two vector in both dimensions is plotted

The instantaneous relative grid altitude $Z_{g}$ corresponding to each snapshot is shown on the sinusoidal plot. Positions are numbered from $p=1$ to $p=5$. Trailing vortices behind grid bars are evidenced again, and their growth and decay can be visualized as a function of the grid position. In DPS, a periodic vortex shedding is observed on both sides of the ROI, specifically in the region where the two mean vortices are formed. The shed vortex are those previously evidenced on Fig. 7 third line.

\subsection{Turbulence, oscillatory motion, and mean flow analysis}

\subsubsection{Reynolds and oscillatory stresses}

To quantify the relative intensity of oscillatory versus turbulent fluctuations in both fluids, the root mean square (rms) over the whole set of instantaneous fields of oscillatory and turbulent fluctuations (regardless of the grid position), defined at Eqs. (16) and (17), respectively, are represented in Fig. 9. The Reynolds and "oscillatory" stresses $\overline{u_{i}^{\prime} u_{j}^{\prime}}$ and $\overline{u_{i}^{*} u_{j}^{*}}$ are shown in Figs. 10 and 11, respectively. In these figures, the grid is sketched at its mean position $(Z=0 \mathrm{~mm})$. The shaded area is not represented since these fields are statistical quantities involving several grid positions. The stresses for which $i=j$ are referred to as "normal" stresses, and the other ones as "tangential" stresses. 
Fig. 7 Ensemble average (first line), phase average for a given grid position (second line), oscillating component for a given grid position (third line) and turbulent fluctuating component example at the same grid position (fourth line) for velocity and vorticity fields, in water (left column) and DPS (right column)

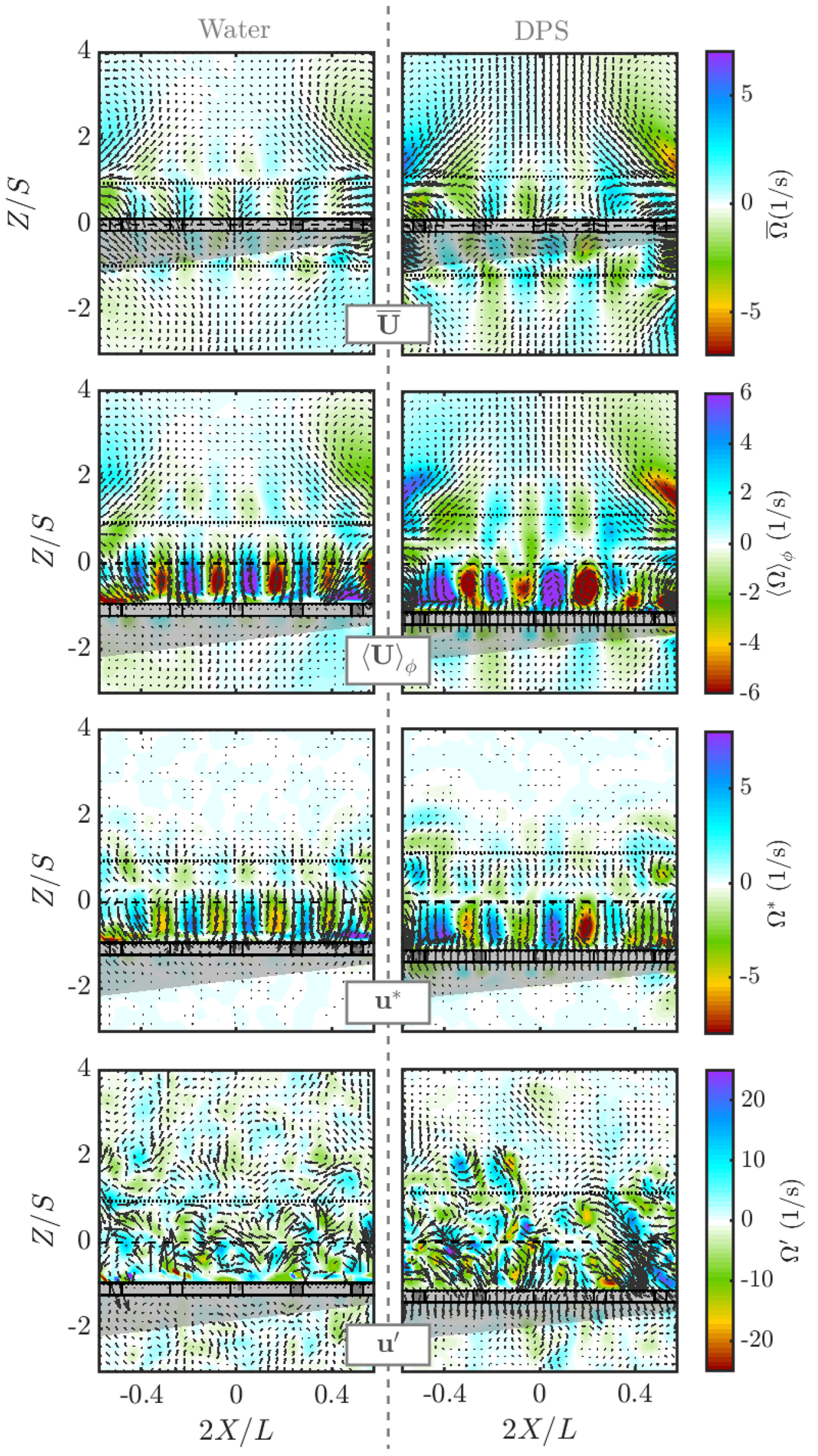



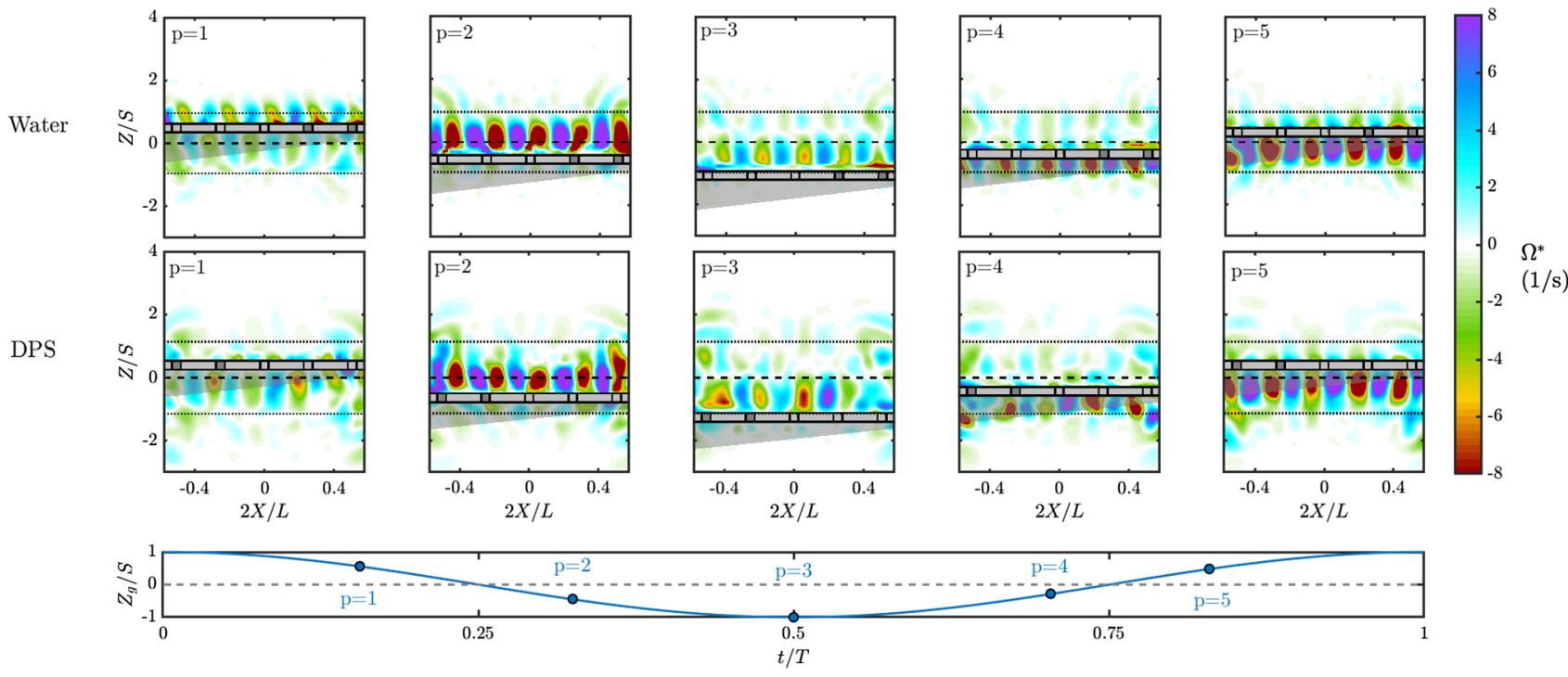

Fig. 8 Oscillating vorticity $\Omega^{*}$ field in $(1 / \mathrm{s})$ at different grid positions in the close grid region for water and DPS. The sampling of the grid period is sketched alongside the figure, on the $Z_{g} / S$ versus $t / T$,

All turbulent fluctuations are logically much higher in the swept region of the grid around $Z=0$. They also exist at a lower level outside of the stroke region in both water and DPS (non zero RMS of turbulent fluctuations). The RMS of the oscillatory component is strong in the grid swept region, as also suggested by the snapshots of typical oscillation period shown in Figs. 7 and 8, but quickly vanishes when moving away from the grid. In DPS, the RMS of oscillatory motion is slightly increased outside of the grid region compared to water. Figure 9 thus shows that the amplitude of oscillatory component of the flow is mostly significant in the swept region of the grid, and quickly becomes negligible compared to turbulence outside of this region. The effect of DPS seems to be an enhancement of the oscillatory fluctuations intensity and a modification of their spatial distribution.

Similar conclusions can be drawn from Figs. 10 and 11: Reynolds stresses and oscillatory stresses are high in the swept region for both water and DPS. They decrease when moving away from this region, and oscillatory stresses rapidly become nil. The presence of polymer tends to increase the spatial decay of Reynolds stresses, but slightly decrease that of oscillatory stresses, especially for the normal horizontal ones. Stress fields yet allow to make a few more observations. First, both the Reynolds and oscillating stresses seem to be increased at $Z=0 \mathrm{~mm}$ in DPS compared to water. Second, the intensity of normal Reynolds stress in DPS is enhanced at the centre of the ROI whereas that of oscillatory stress is larger on the sides of the ROI. Finally, the two side vortices evidenced from the mean flow study are found to correspond to two patches of tangential Reynolds where $Z_{g}$ is the distance from the grid to its mean position, and $t$ the time over a grid period $T$

stress with a significant magnitude, and that for both water and DPS, as visualized on Fig. 10 second row.

\subsubsection{Energy transfers}

The transfer terms have been defined in Sect. 2.3, Eqs. (5), (6) and (7). The $\mathscr{T}_{\text {mt }}$ (mean to turbulence), $\mathscr{T}_{\text {mo }}$ (mean to oscillatory) and $\mathscr{T}_{\text {ot }}$ (oscillatory to turbulence) fields for water and DPS are shown in Fig. 12. Noticing the scales of each color bar, one sees that of magnitude, $\left|\mathscr{T}_{\mathrm{mt}}\right|<\left|\mathscr{T}_{\text {mo }}\right|$, $\left|\mathscr{T}_{\mathrm{mt}}\right|<\left|\mathscr{T}_{\text {ot }}\right|$, and $\left|\mathscr{T}_{\text {mol }}\right| \approx\left|\mathscr{T}_{\text {ot }}\right|$. In water it appears that $\left|\mathscr{T}_{\text {mo }}\right|<\left|\mathscr{T}_{\text {ot }}\right|$, but in the polymer case $\left|\mathscr{T}_{\text {mo }}\right|>\left|\mathscr{T}_{\text {ot }}\right|$.

From the topology of these fields, several observations can be made:

- The variations of transfer terms are larger in DPS than in water. Since transfer terms are a product of stress and spatial gradient, the origin of these variations may come from both of these parameters. Reynolds and oscillatory stresses spatial variations are more important in DPS than in water (see Figs. 10, 11). Mean and oscillatory flows are enhanced by the presence of polymer (as shown in Fig. 7), and their gradients are sharper.

- All the transfers mostly occur within the grid swept region. The only small but noticeable exception is observed for $\mathscr{T}_{\mathrm{mt}}$ fields in DPS, just above the grid's top position, where two weak mean to turbulence transfer regions are observed on both sides of the ROI (evidenced by dashed circles on Fig. 12, first line of sub-figures). They correspond to the mean flow vortices observed pre- 
Fig. 9 RMS of oscillatory (lines 1 and 2) and turbulent (lines 3 and 4) velocity fluctuations along the horizontal (lines 1 and 3 ) and vertical (lines 2 and 4) directions, in water and DPS. Velocities are expressed in $\mathrm{m} / \mathrm{s}$ and displayed in log scale. Isovalue lines are drawn in white full lines at $\log$ values of $-3,-2.5,-2,-1.5$ and -1 on each sub-figure
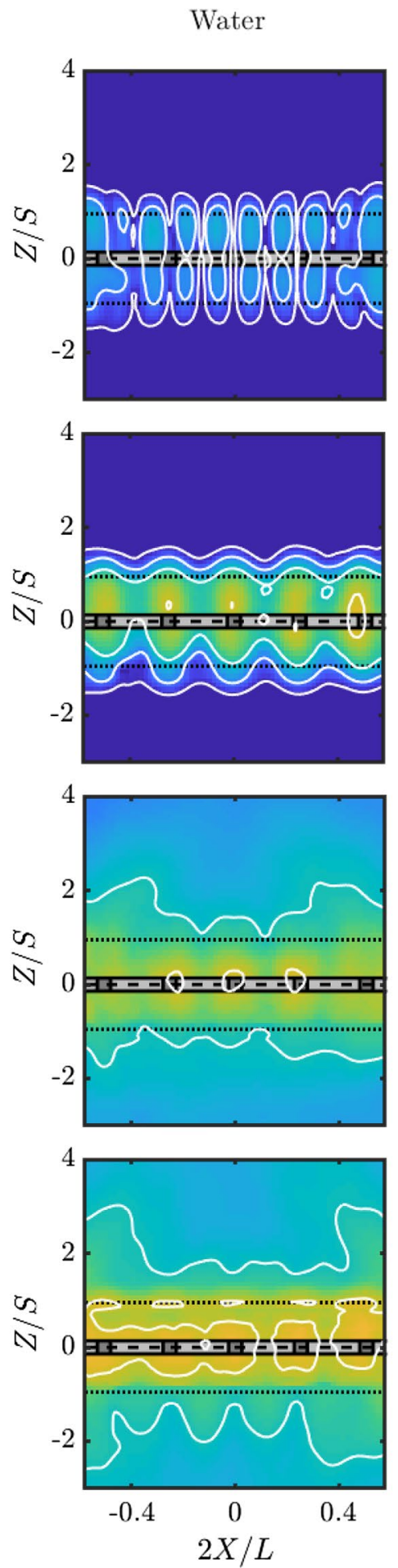

DPS
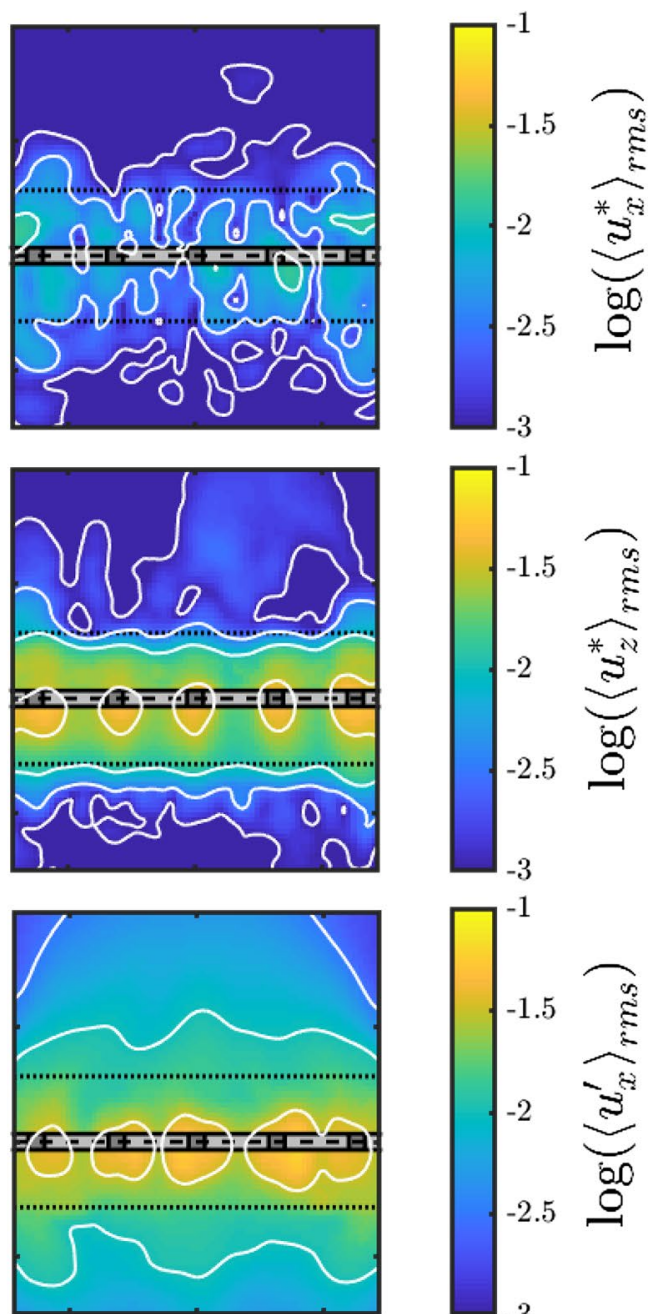

$-3$
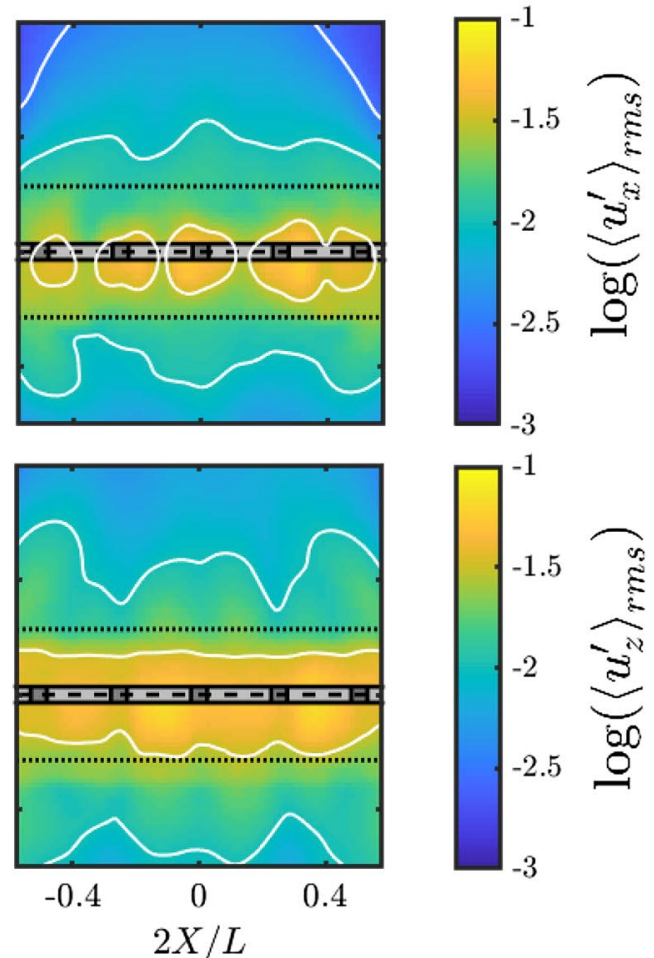

viously. This is the blueprint of a secondary turbulence production region, the strength of which is enhanced by the presence of polymer. It was also evidenced by the enhancement of $\overline{u_{x}^{\prime} u_{z}^{\prime}}$ Reynolds stresses in the same region in Fig. 10.

- $\mathscr{T}_{\text {mo }}$ seems stronger in the DPS than in the water case, as illustrated by the second row of the figure. This underlines the increased importance of the oscillatory field in DPS. In particular, two highly negative $\mathscr{T}_{\text {mo }}$ patches appear on the top part of the swept zones at the sides of the ROI (hence near the walls). Negative $\mathscr{T}_{\text {mo }}$ means that energy is transferred from the oscillatory motion to the mean flow. The location of this patches corresponds quite well to that of enhanced mean flow 
Fig. 10 Reynolds stresses in water and DPS. Isovalue lines are plotted in full or dasheddotted lines and their values are indicated by arrows on the figure
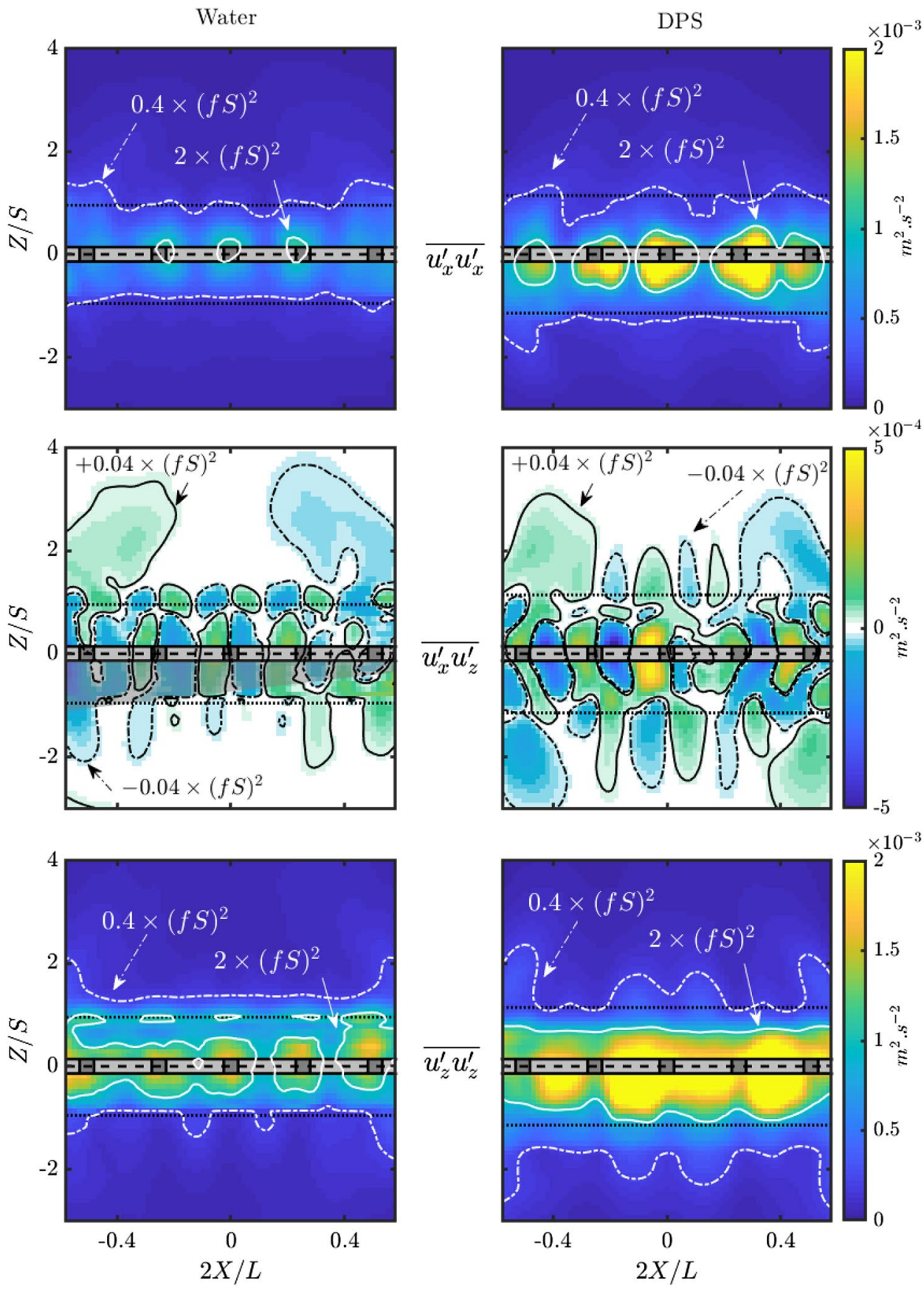

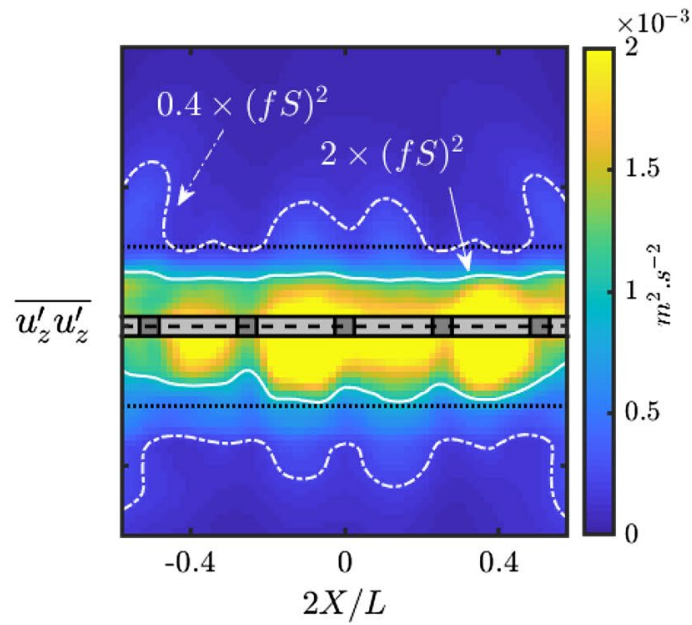

structures observed in Fig. 7 first line. Energy transfer terms thus bring the additional information that this mean flow enhancement comes from the mean flow production by oscillatory flow at the walls.

- In water, $\mathscr{T}_{\text {ot }}$ mostly occurs in vertical regions aligned with the grid bars, and the transfer term is always positive, so that oscillatory motion seems to always transfer energy to turbulence and not the other way around. In DPS, the pattern of the $\mathscr{T}_{\text {ot }}$ field is more elaborated and both positive and negative regions exist, which means that energy transfer is a two-way process. This is con- sistent with the two-way flow-polymer interaction process evidenced by Nguyen et al. (2016) in viscoelastic turbulence.

Figure 13 summarizes Figs. 10, 11 and 12 in terms of vertical profiles of Reynolds and oscillatory stresses and transfer terms. All curves have been obtained by width averaging the corresponding fields over the $-50<X<50 \mathrm{~mm}$ range. Only the values for $Z>0$ are shown.

These curves confirm and complete the earlier observations. Oscillatory and Reynolds stresses are maximum in 
Fig. 11 Oscillatory stresses fields induced by the periodic velocity fluctuations in water and DPS. Isovalue lines are plotted in full or dashed-dotted lines and their values are indicated by arrows on the figure
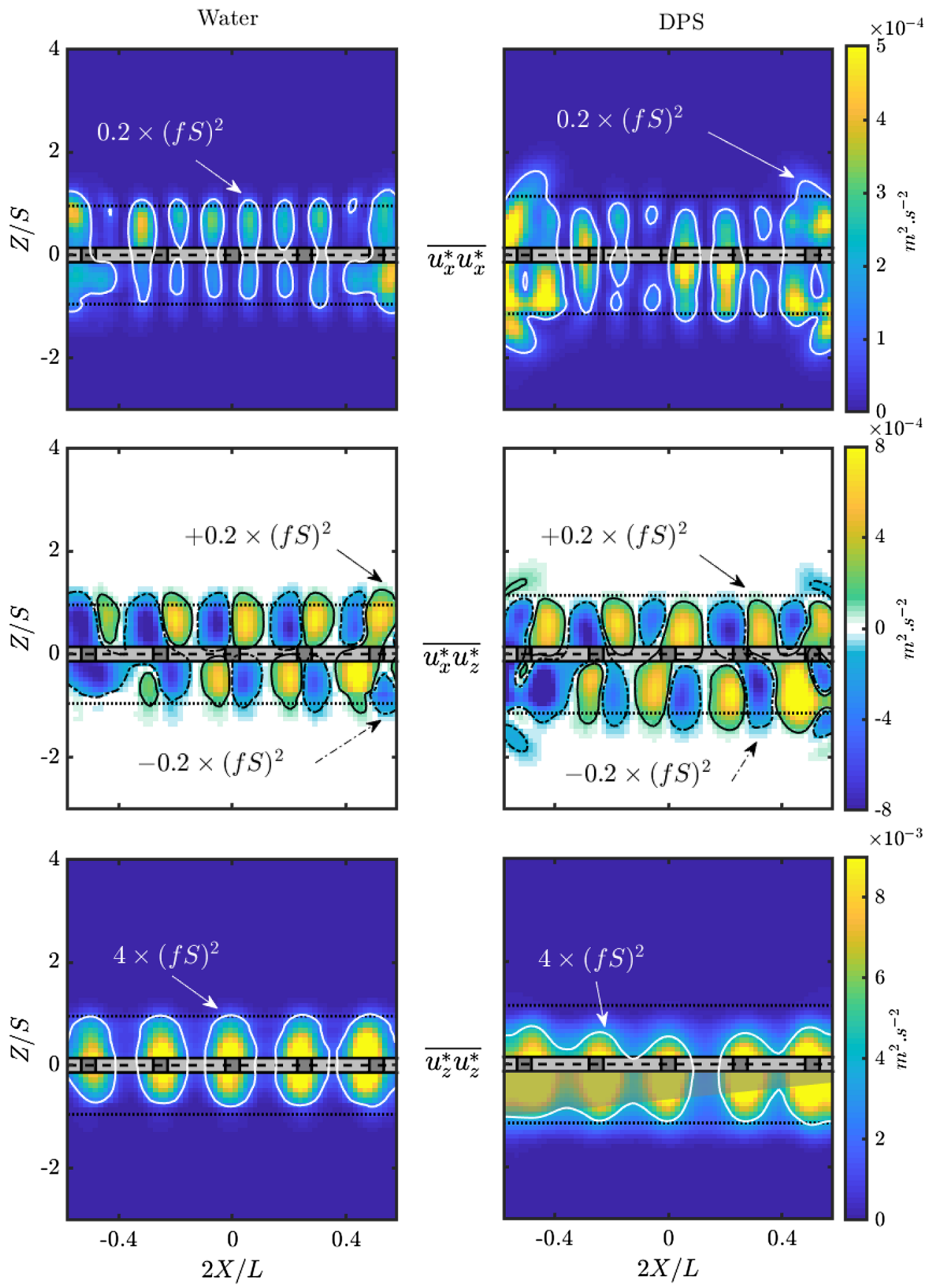

the core of the grid swept region, close to $Z=0$. Oscillatory stresses decay much faster than the Reynolds stresses with increasing $Z$, and are negligible compared to the Reynolds stresses outside of the swept region, by at least a factor 10 . For a given fluid and a given type of fluctuations, the vertical stress is always larger than the horizontal one. This is obviously because of the vertical grid displacement. This effect is all the more important for the oscillatory stresses which are directly due to this grid motion. The presence of polymer slightly increases the Reynolds stress value at the origin, but also the decay rate of the stresses with $Z$. In other words,
DPS tends to concentrate the effects of turbulence in the swept region. As for the effect of polymer on the oscillatory component, it was previously observed that DPS solutions showed enhanced oscillatory structures outside of the swept region on the sides of the ROI. However, this effect was found to be related to tangential stresses and not to normal ones, hence it is not visible on the $\overline{u_{i}^{*} u_{i}^{*}}$ fields and on their vertical profiles.

As for energy transfers, the vertical variations of transfer terms is increased in DPS as compared to water in the swept region. This is supposedly due to both the enhancement of 
Fig. 12 Triple decomposition transfer terms $\mathscr{T}_{\text {mt }}$ (mean to turbulent), $\mathscr{T}_{\text {mo }}$ (mean to oscillatory or periodic) and $\mathscr{T}_{\text {ot }}$ (oscillatory to turbulent) in water and DPS. Transfer terms are expressed in $\mathrm{m}^{2} \mathrm{~s}^{-3}$. Isovalue lines are plotted in full or dashed-dotted lines and their values are indicated by arrows on the figure
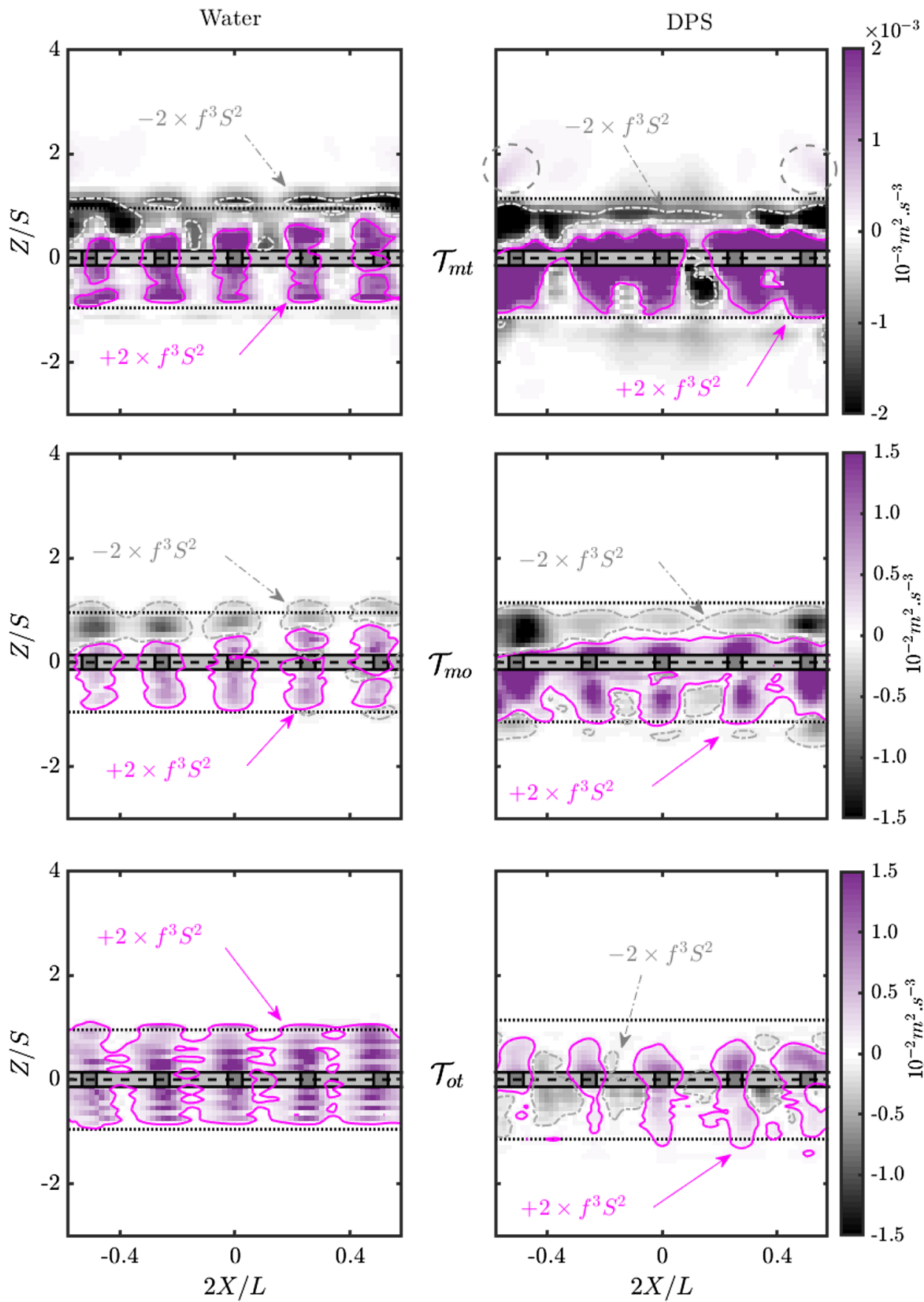

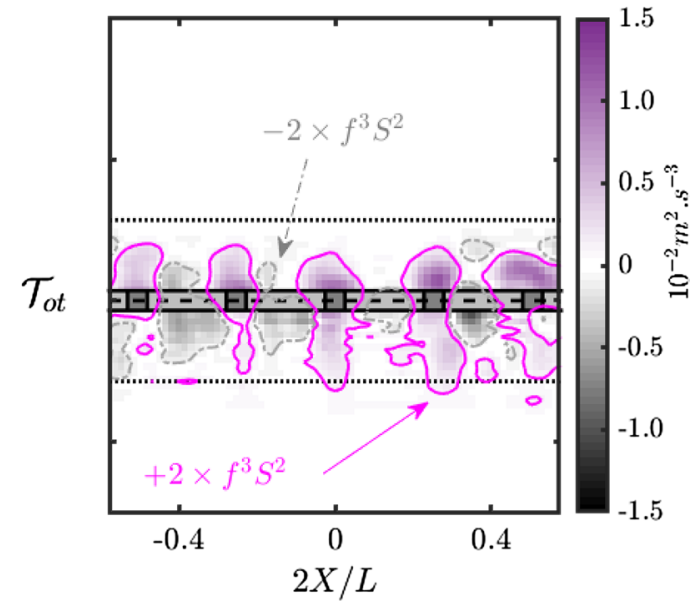

oscillatory and Reynolds stresses and to the spatial gradients of mean and oscillatory velocities. Such results suggest that gradient-based models could be good candidates at closure relationships for the estimation of these transfer terms. In the core of the swept region, all width-averaged transfer terms are positive for water and DPS: energy is transferred from the mean and oscillatory flows to turbulence. At the edge of the grid swept zone, $\mathscr{T}_{\text {mo }}$ and $\mathscr{T}_{\text {mt }}$ profiles become negative suggesting a feeding of the mean flow, that is further enhanced in DPS.
An important conclusion of the present work is thus that, outside of the grid swept zone, oscillatory, grid correlated, fluctuations quickly vanish in front of random turbulent ones with increasing Z/S. Despite its enhancement upon polymer addition previously mentioned, the oscillatory motion's intensity (quantified by the rms of oscillatory fluctuations, Fig. 9, or by oscillatory stresses, Fig. 11) is one to several orders of magnitude lower than turbulence intensity. This legitimates the use of the classical Reynolds decomposition outside the grid swept zone when working in a fixed, tankattached reference frame (as done in Lacassagne et al. (2019) 

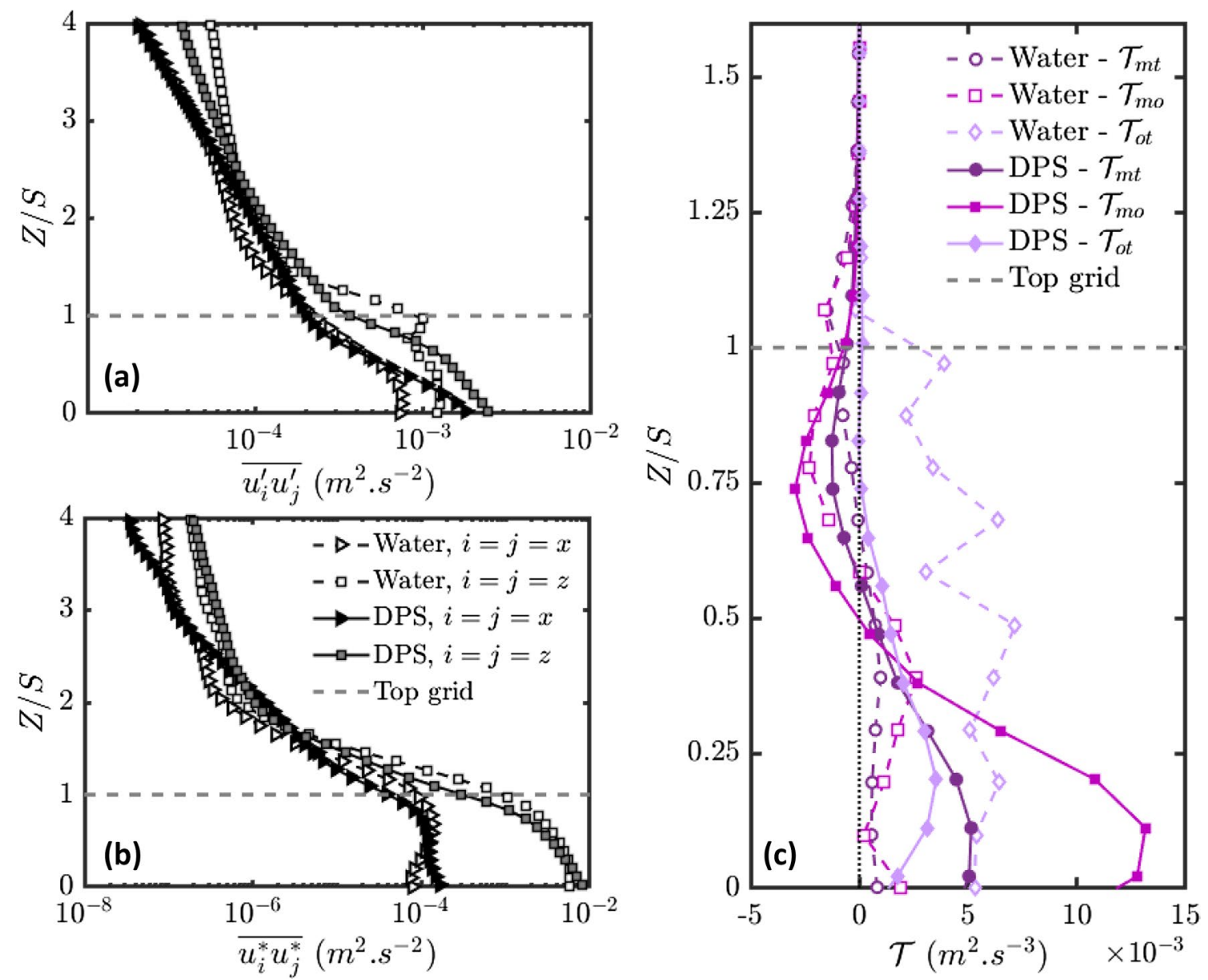

Fig. 13 Vertical profiles (width-averaged over the ROI) of a Reynolds normal stresses, $\mathbf{b}$ oscillatory normal stresses, $\mathbf{c}$ transfer terms, for water and DPS

since the oscillatory fluctuations can be assumed negligible there. However, it must be kept in mind that any study presenting turbulence properties inside the swept zone from a classical Reynolds decomposition implicitly includes oscillatory motion into either mean flow or turbulent fluctuations.

\subsubsection{About mean flow generation}

One of the key questions regarding OGT is the existence of mean flows, often an unwanted feature of the device. Its origin is most of the time attributed to misalignment or default in symmetry between the grid and the tank (McKenna and McGillis 2004a). McCorquodale and Munro (2018b) based on the older work by Drayton (1993) advance a possible source for the establishment of mean flow in the tank. They state that mean flow is induced in OGT when there is a significant difference in the relative strength of the jets produced by the oscillating grid in different regions of the tank, and suggested the Coanda effect (applied on jets closest to the tank's wall) to be the dominant mechanism for this.
Indeed as argued by Drayton (1993), jets close to the wall are necessarily non symmetric fashion, their growth being inhibited in the wall-normal direction. Compared to central jets, they should spread at higher grid distances in the walltangential, grid-normal direction, and consequently be less likely to merge with their neighbours. They are thus more likely to reach the outside of the grid swept zone and to give rise to a persistent flow on the sides of the tanks, resulting in the mean recirculation structures observed in several studies (Drayton 1993; Lacassagne et al. 2019; McCorquodale and Munro 2018b; McKenna and McGillis 2004a).

The previous description applies quite well in the present case, for both water and DPS: Fig. 6, 7 and 8 show that the side jets at $|2 X / L|>0.4$ are significantly non symmetrical compared to central jets. This is correlated to persistent mean flow structures on the sides of the ROI at simi$\operatorname{lar}|2 X / L|$ values (Fig. 7, first line). Moreover, Fig. 9, lines 3 and 4, shows that higher turbulent velocity rms values are reached in the trails of grid bars at the centre of the ROI, compared to grid bars closer to the wall. The relative 
variation of jet strengths (in addition to the asymmetry) is thus also confirmed.

One striking results is that the addition of polymer seems to enhance the intensity of the mean flow. As previously mentioned, side vorticities seem stronger in DPS than in water, and accompanied in DPS only by an enhanced up-going motion above the swept zone. Looking back at Figs. 6, 7, 8 and 9, one sees that all the features characteristic of mean flow production as described by McCorquodale and Munro (2018b) and Drayton (1993) are even more obvious and enhanced in DPS compared to water. Asymmetry of the side jets is enhanced, and jets at the centre of the tank have even higher intensities with respect to the side ones when considering rms fields (Fig. 9).

The description of the mechanisms of mean flow production by Drayton (1993) thus seems to hold in water and DPS since an enhancement of jet asymmetry and intensity variations leads to a stronger mean flow. This yet does not explain why asymmetry and relative intensity variation is enhanced in DPS. We infer that in DPS, the Coanda effect should also be accompanied by shear-thinning variable viscosity actions. Indeed, jets close to the walls are in a region for which the fluid is submitted to a sustained high shear rate. The imposed velocity gradient is strong since the grid forces a periodic velocity, the velocity at the wall is nil and the variations between the two boundary constrained velocities happen within the very small gap between the grid and the wall (typically $1 \mathrm{~mm}$ here between the edge of a grid bar and the wall). Since the shear rate is high, viscosity should be low in this region. However when moving away from the walls, the imposed periodic shear rate is decreased and viscosity thus increases. The consequence is that side jets are inherently coupled with an horizontal viscosity gradient, increasing their asymmetry and the non horizontally homogeneous repartition of intensities in the jets. Such a behavior would thus tend to enhance mean flows as described by Drayton (1993) and McCorquodale and Munro (2018b).

Additional information can be brought to this mechanism by the present data, regarding the distance from the grid at which mean flow enhancement occurs. Indeed, Fig. 13 shows the profiles of width-averaged energy transfer terms $\mathscr{T}_{\text {mo }}, \mathscr{T}_{\mathrm{mt}}$ and $\mathscr{T}_{\mathrm{ot}}$. Considering that the energy inputs, by the grid, are made towards the oscillatory component, mean flow production should thus be a consequence of energy transfers from oscillatory motion to mean flow, evidenced by negative $\mathscr{T}_{\text {mo }}$ values. This is achieved in the $0.5<Z / S<1$ region, i.e., inside but at the outer part of the grid swept zone in both water and DPS. In the DPS case, the $\mathscr{T}_{\text {mo }}$ reaches more negative values, confirming and enhanced mean flow production. Moreover, one notices that in the water case, the $\mathscr{T}_{\text {ot }}$ term is always positive, and significantly higher that in the DPS case. This means that in water, most of the energy input at the oscillatory level is used to generate turbulence, and only a smaller part of it creates mean flow: $\left|\mathscr{T}_{\text {ot }} / \mathscr{T}_{\text {mo }}\right|$ is typically equal to 1.5 at $Z / S=0.75$. In DPS, a more significant part of the oscillatory energy is transferred to the mean flow: $\left|\mathscr{T}_{\text {ot }} / \mathscr{T}_{\text {mo }}\right|$ falls to 0.03 at the same altitude. ${ }^{2}$

\subsection{Phase turbulence}

Another way to describe the periodicity of turbulence property is to compute rms values of velocity fluctuations not on the full set of data, but for each specific grid position. Such so-called phase rms $\left\langle u_{z}^{\prime}\right\rangle_{\phi, \mathrm{rms}}^{k}$ are defined at Eq. 15. An example of phase rms field for water for a given grid position is given in Fig. 14a, the log-scaled color bar representing the magnitude of $\left\langle u_{z}^{\prime}\right\rangle_{\phi, \mathrm{rms}}^{k=p=1}$ in $\mathrm{m} / \mathrm{s}$. One can clearly evidence the wake of the grid by high turbulent intensity patches. We should stress that the following analysis is not performed in the global Eulerian frame of the tank, for which oscillatory flow has already been considered through non-phased rms of oscillatory fluctuations, or oscillatory stress fields. This time, we are looking at oscillatory flow in a grid-attached Eulerian frame: the oscillatory aspect of the flow is intrinsically accounted for by defining turbulence based on the grid position.

It is interesting to notice that the higher turbulent intensity regions are found to correspond to grid holes. Previously, it was observed that normal Reynolds stresses (Fig. 10) and non-phased rms fields (Fig. 9) where maximal above grid bars. Tangential Reynolds stresses were themselves maximal above grid holes. Here we understand that for a given grid position, turbulence is high where the wakes of grid bars meet, i.e., in the jets behind the holes. In this region, both vertical and horizontal fluctuations are found since structures are the result of a lateral collision of structures, advected by the oscillatory trailing vortices shown in Fig. 8. This thus explains the mapping of tangential Reynolds stresses. Vertical Reynolds stresses, as for rms of $u_{z}^{\prime}$, are maximal in the region where turbulence production is the result of vertical sweeping by bars only.

In the rest of this paragraph, five different sampling grid positions are considered (already used in Fig. 8 for the visualization of a typical vorticity phase). They are represented on a grid period in time and space in Fig. 14b). Position 1 correspond to a grid accelerating downwards, position 2 to a grid still going downwards, but decelerating. Position 3 is the extreme low position at $Z=-S$, where the velocity is nil and the acceleration maximal and oriented upwards. Positions 4 and 5 are for the grid going upwards and respectively accelerating and decelerating. For each grid sampling

\footnotetext{
2 The consequence of this enhancement in terms of mean flow properties is described in Lacassagne et al. (2019), for various polymer concentrations.
} 
Fig. 14 Phase rms of velocity fluctuations. a Phase rms field for the vertical velocity fluctuation $\left\langle u_{z}^{\prime}\right\rangle_{\phi, \text { rms }}^{k=p}$ at sketch position index $p=1$, in log color scale. b Sketch of the sampled grid positions numbered 1-5. c, d Profiles of phase rms plotted as a function of the instantaneous grid distance normalized by the grid stroke $\left.Z-Z_{g}\right) / S$ for respectively water and DPS, at the five sampled positions sketched on sub-figure b. The dashed black line and dasheddotte gray line illustrate, respectively $\left(Z-Z_{g}\right)^{-1}$ and $\left(Z-Z_{g}\right)^{-2}$ trends. Dashed color lines on sub-figure $\mathbf{c}$ are least square fittings of $p=1$ and $p=2$ curves by power laws. The grayed region represents half the width of the grid bar along $\left(Z-Z_{g}\right) / S$
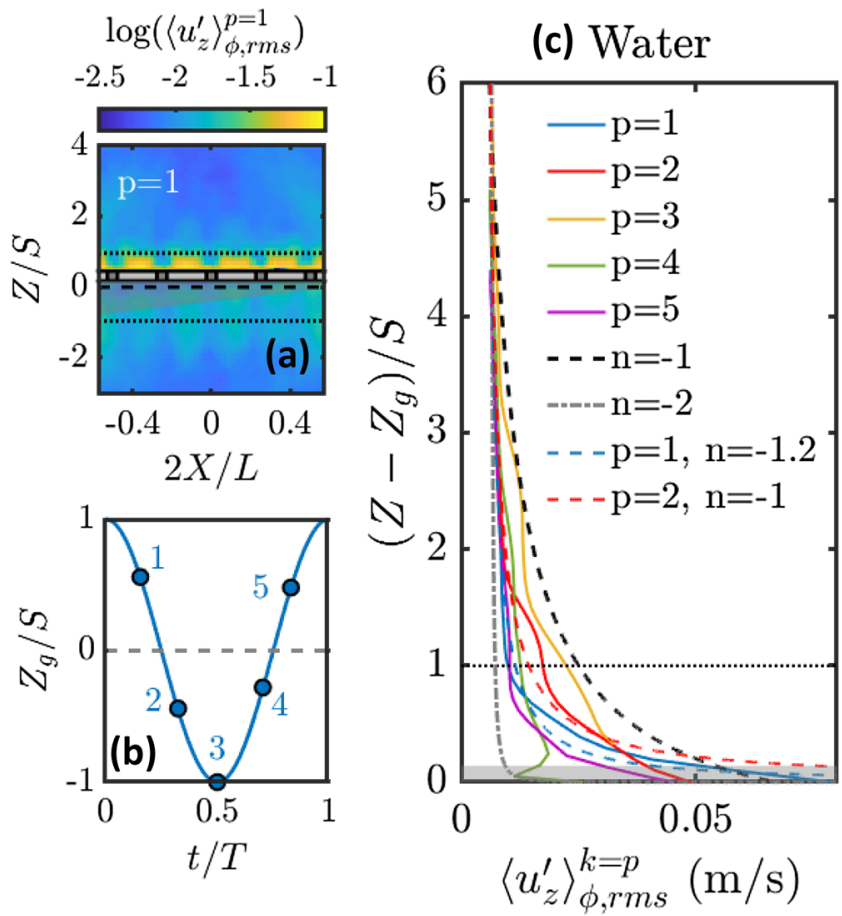

(d) DPS

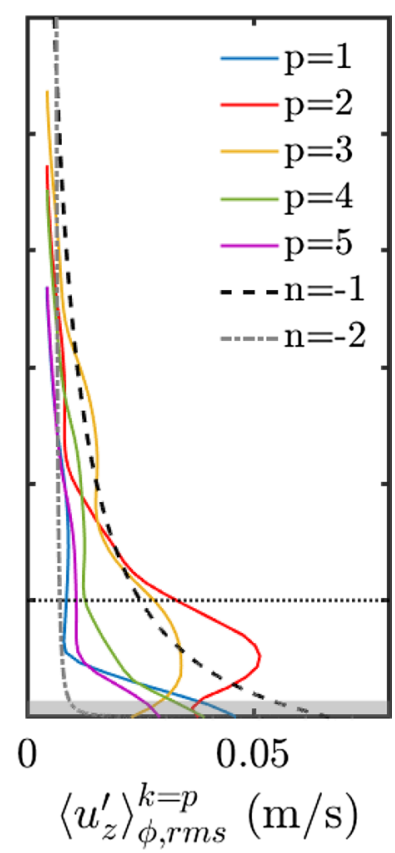

position $k=p$, phase rms fields are averaged over the horizontal dimension $X$. The width-averaged profiles of $\left\langle u_{z}^{\prime}\right\rangle_{\phi, \text { rms }}^{k=p}$ are plotted versus the instantaneous distance from the grid $Z-Z_{g}$ normalized by $S$ in Fig. 14c (water) and d (DPS).

For almost all grid positions and for the two fluids considered (except $p=2$ and $p=3$ in DPS as discussed later), rms profiles roughly follow power law decay trends such that $\left\langle u_{z}^{\prime}\right\rangle_{\phi, \mathrm{rms}}^{p} \sim\left(Z-Z_{g}\right)^{n}$. All curves are included inside an envelope defined by $n=-2$ and $n=-1$ trends. The $n=-1$ value happens to be the usual exponents of the decay law for oscillating grid turbulence evidenced by Hopfinger and Toly (1976) when plotting non phased rms profiles of $\left\langle u_{z}^{\prime}\right\rangle_{\mathrm{rms}}$ as a function of the dimensional $Z$. We see that here, once phased to the grid motion, the decay profiles of each grid positions has a $n$ value lower than -1 , but higher than -2 .

In water, one could probably expect that when phasing turbulence with the grid position, the decay exponents characteristic of fixed grid turbulence could be retrieved for well chosen grid positions. Indeed, one can consider an Eulerian description on the flow in a reference frame attached to the grid and no longer to the tank. When the grid is going down (respectively up), the configuration for each grid position that of a fixed grid with flow passing through in the $+Z$ (respectively, $-Z$ direction), with a relative fluid velocity equal to the grid's velocity. For fixed grids, Comte-Bellot and Corrsin (1966) and many more recent works (listed in Hearst (2015) evidenced decay exponents for the turbulent kinetic energy in the $[-1.4,-1]$ range. For the streamwise

velocity fluctuations considered here, this should yield decay exponents in the $[-1.2,-1]$ range. Here curves for position $p=1$ and $p=2$ in Fig. $14 \mathrm{c}$ correspond to the situation analog to that of a fluid flowing upwards through a fixed grid, and should be comparable to fixed grid scaling laws. They are fitted by expressions of type $\left\langle u_{z}^{\prime}\right\rangle_{\mathrm{rms}}=a \times\left(Z-Z_{g}\right)^{n}$. Values of $n$ obtained for $p=1$ and $p=2$ are, respectively, equal to -1.2 and -1 , with a better fitting for $p=1$.

This phase analysis suggests that original mechanisms of turbulence generation by the oscillating grid are in fact quite comparable to those behind a fixed grid in a mean flow, at least as a first approximation: a grid moving in a fluid quite logically generated turbulence in a similar fashion that when a fluid moves across a fixed grid. Yet the fundamental difference between the two setups is that turbulent structures generated at previous grid positions are still present in the flow at following grid positions and may interact with the newly generated structures. This is one of the possible reason why the fitting for the $p=2$ position is less efficient, and why the decay exponent varies between -1.2 and -1 . Indeed, for $p=1$ most of the $Z-Z_{g}>0$ region lays outside of the grid swept zone, while for $p=2$, low $Z-Z_{g}$ values are found inside this grid swept zone, when pre-existing turbulent structures interact with the $p=2$ jets and wakes.

All of the above is valid for water. When considering DPS (Fig. 14d), the first observation is that profiles no longer follow single exponent power law trends, at least in the vicinity of the grid. For $p=1$ and $p=5$, a two-slope trend is visible with a change of slope at $\left(Z-Z_{g}\right) / S \simeq 0.5$. For $p=2$ and $p=3$, a peak of $\left\langle u_{z}^{\prime}\right\rangle_{\mathrm{rms}}$ is observed at the same scaled 
distance. This shift in decay rate concerns top grid positions, and evidences a boundary between high turbulent intensity, near-grid region and the rest of the flow above the swept zone that for grid positions. The second behavior, namely the rms peak, is characteristic of a turbulent patch shedding by the grid: at bottom grid positions $(p=2,3)$ and with a grid going down, previously generated turbulent structures are advected away from the grid by the hole jets. Since the grid is slowing down ( $p=2$ ) or even stopping ( $p=3$ ), turbulence generation is less efficient and up-going turbulent patches break away from the near-grid region. This is no longer observed for $(p=4)$ since the grid is going up and catches back on the shed turbulent patches. We thus see again an enhanced importance of flow periodicity in term of turbulence generation for the DPS case.

It is worth mentioning that this phase rms study can be another way of describing the periodicity of the flow induced by the grid. It is here addressed not in terms of periodic flow structures produced, but as a periodic behavior of turbulent properties. Figure 14 shows that if this periodicity of turbulence is significant in the swept zone (see sub-figure a as an illustration), it reduces when moving away from the grid, as evidenced by the asymptotic collapse of all curves for $\left(Z-Z_{g}\right) / S>3$. This seems to indicate that outside of the grid swept zone, turbulent properties are also not periodic. However, clearer evidence could be brought by performing time resolved measurement of turbulence generated by the grid and studying the temporal periodicity of correlation functions of turbulent correlations to themselves (or turbulent timescales)in the Eulerian tank-attached frame.

\subsection{Viscosity estimation and statistics}

A fast approach for studying polymer influence on the origins of OGT is an estimation of the local viscosity field and its statistics. The main idea is to derive local shear rate fields from instantaneous, phase-averaged or time-averaged velocity fields. Local shear rate values are then used to estimate an apparent viscosity through the constitutive equation of the polymer, here a CY law for the rheological behavior derived in Sect. 3.1 (Eq. 8, Fig. 2).

This method is limited because the velocity data is only 2D and lacks the third velocity component. Shear rate values can thus only roughly approach the real local shear rate, and no precise quantitative study can be performed. However, the method allows to qualitatively estimate the viscosity variations in a plane of measurement.

In practice, the apparent instantaneous shear rate is computed as

$\dot{\gamma}_{\mathrm{app}}=\sqrt{\left(\frac{\partial U_{x}}{\partial z}\right)^{2}+\left(\frac{\partial U_{z}}{\partial x}\right)^{2}}$, and the instantaneous apparent viscosity as

$\mu_{\text {app }}=f_{\mathrm{CY}}\left(\dot{\gamma}_{\text {app }}\right)$

with $f_{\mathrm{CY}}$ the Carreau-Yasuda function defined in Eq. (8), and using fitting parameters obtained in Sect. 3.1. Statistics for viscosity are then obtained by ensemble averaging over the whole set of field for $\bar{\mu}_{\text {app }}$, or grid position by grid position for $\mu_{\text {app }}^{*}$ exactly like what is done for the velocity field.

Figure 15 shows the fields of ensemble average viscosity $\bar{\mu}_{\text {app }}$, ensemble rms of turbulent viscosity fluctuations

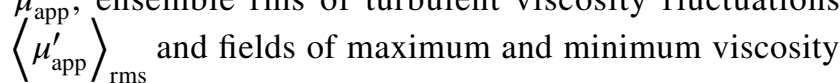
values over time $\mu_{\text {app,max }}$ and $\mu_{\text {app,min }}$. All values are scaled by the $\mu_{0}$ maximum viscosity.

Estimating the uncertainty on instantaneous viscosity field is quite arduous since one would need to know the uncertainty on instantaneous velocity fields and instantaneous local shear rates. However, another approach can be used to evaluate the error associated to this viscosity estimation process. From statistical convergence analysis (see Fig. 5), we estimate the uncertainty on velocity statistics convergence to be at worst of $5 \%$. Uncertainty propagation on velocity gradient and shear rates can be performed to quantify the resulting relative uncertainty on apparent shear rate, which may be up to $30 \%$. From this value, the CY law (Eq. 8, Fig. 2) can finally be used to assess uncertainty on calculated apparent viscosity at each shear rate value. This last uncertainty is maximal when the slope of the curve in Fig. 2 is the steepest, i.e., at the lowest effective flow index (as defined in Cagney and Balabani (2019)). This is here the case for shear rates around $4 s^{-1}$. At this worst case shear rate, the relative uncertainty of apparent viscosity is found to be $14 \%$.

The first sub-figure of Fig. 15 shows that average viscosity is minimal close to $Z=0$ inside the swept grid region. It then increases when moving away from $Z=0$ until it reaches its zero shear rate plateau value at $Z / S>3$. The maximum of rms velocity fluctuations is found just above the grid's top and bottom positions, around $X=0$. Here the viscosity varies from a value close to its zero shear rate plateau when the grid is on the other side of its stroke, to a much lower value when the grid departs from this position and releases high shearing eddies. Vertical trails of high viscosity rms are also observed in regions associated to grid holes. Since this plot represents the rms of turbulent viscosity fluctuations, it means that these trails are not due to oscillatory motion, but rather to strong fluctuations of the shear rate fields because of turbulent structures in those regions. The central high viscosity fluctuation path in the $X=0 \mathrm{~mm}, Z / S=1.5$ region confirms the existence of preferential paths for turbulence. Turbulent fluctuations are more likely to be found at the centre of the ROI above the swept zone. The minimum viscosity fields shows that 

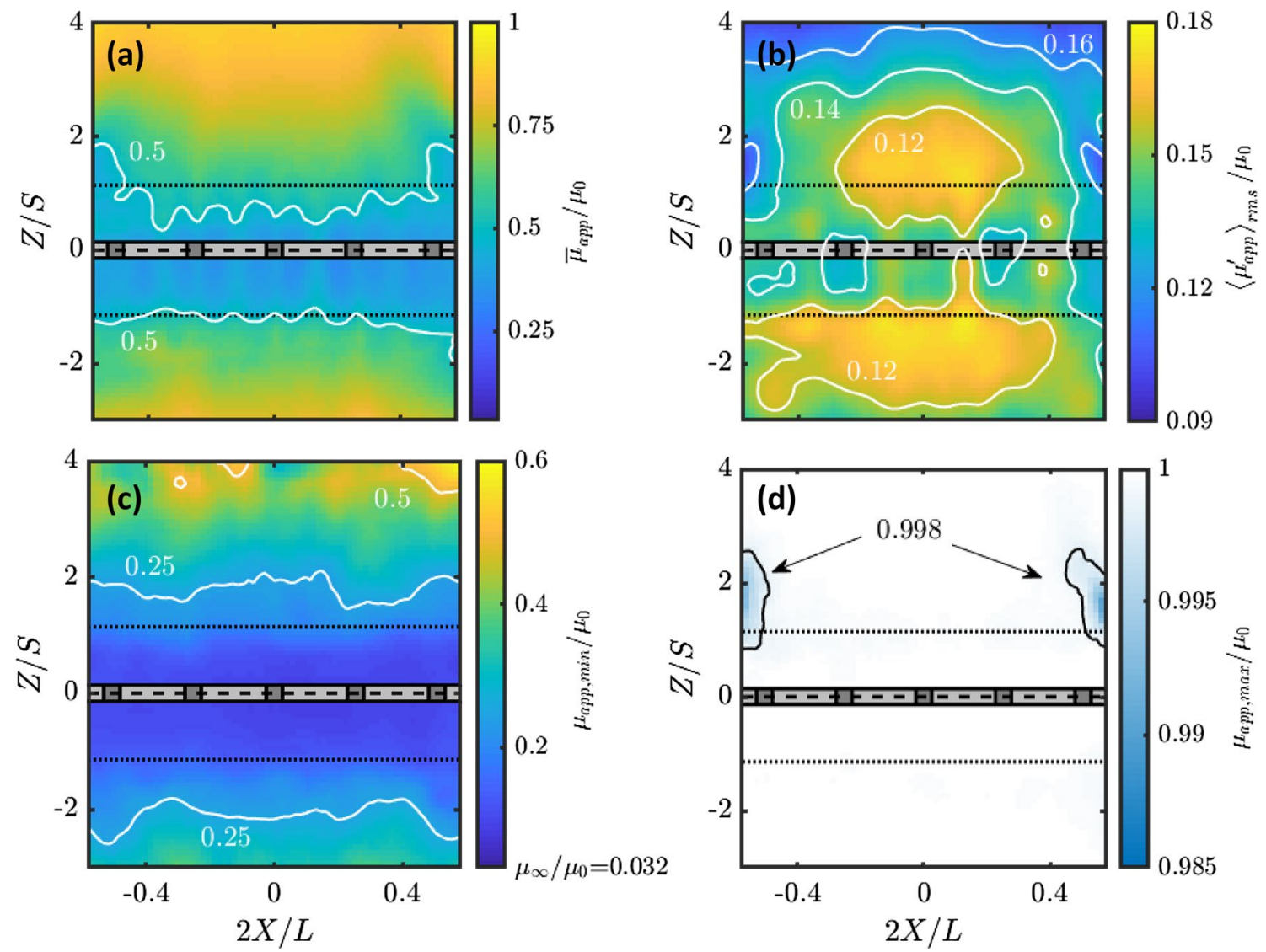

Fig. 15 Local statistics of apparent viscosity: average (a), rms of turbulent fluctuations (b), minimum (c) and maximum (d). The zero shear rate viscosity in $100 \mathrm{ppm}$ XG solutions is $32.78 \mathrm{mPa}$ s and the

viscosity is always higher than the infinite shear rate viscosity, which means that during the experiments, no shear rate (mean, oscillatory or turbulent) is high enough for the fluid to behave like a low viscosity Newtonian fluid, even locally. Finally, the maximum viscosity field shows that almost everywhere in the fluid, the maximum viscosity value may be reached at a time, i.e., that almost everywhere in the fluid, at some time, the shear rate is almost nil. The only locations where this is not the case are just above the grid swept region. Here lower maximum viscosity patches are found which correspond to the shape and location of the mean flow vortices.

\section{Conclusion}

In this last section, the main conclusions are summarized, first the key features of the flow around a grid oscillating in water are described. The effects of polymer addition are summarized in a second paragraph. Finally the generalization of the present results and perspectives for future works are discussed.

infinite shear rate viscosity is $1.05 \mathrm{mPa}$ s. Isovalue lines are represented by full lines on each sub-figures, with values indicated nearby

\subsection{OGT in water}

The grid periodic motions create oscillatory vortices behind the grid bars. At the walls (on both sides of the ROI), these periodic, non symmetric vortices feed the large mean flow eddies. Oscillatory component is strong within the grid swept region, but quickly vanishes when moving away from the grid's action. This suggests that a classical Reynolds decomposition can equally be used to describe turbulence properties in the bulk flow. Turbulence is created mostly by oscillatory flows in the swept region. It is highly anisotropic, with a much higher intensity along the vertical direction, as imposed by the grid motion. When turbulent intensity is computed in phase with the grid position, its decay law with the distance to the grid can be compared to fixed-grid correlations for well chosen grid positions. The global mechanisms of turbulence and mean flow production by the oscillating grid is the following: the grid introduces turbulence in the oscillatory fields, which transfers energy to the mean flow and to turbulence. 


\subsection{Effects of polymer addition}

Polymer addition has several effects on the flow structure and energy exchanges around the grid. The first one is an enhancement of the oscillatory flow on the sides of the ROI near the walls. This leads to a periodic vortex shedding that feeds the mean recirculation and increase its intensity, thus possibly explaining the mean flow enhancement. The second effects is a modification of the spatial distribution of turbulence. Reynolds stress and turbulence intensity are increased in the core of the swept region close to $Z=0$, and decrease quicker with increasing $\mathrm{Z}$ magnitude. Turbulence is enhanced at the centre of the ROI (far from the walls), both in the swept region and far from the grid. Finally, the transfer from oscillatory to turbulent fluctuations seems more complex in DPS than in water, and suggests that oscillatory motion may locally both give and take energy from turbulence, which was not the case for water. Another visualization of the same phenomenon was made possible by an estimation of viscosity from PIV measurements and its statistical analysis.

\subsection{Perspectives}

It is worth reminding that a limitation of the previous analysis is the fact measurements are planar and do not account for the highly three-dimensional behavior of turbulence. Results may be different when considering for example a plane of measurement not coincident with a grid bar, but with a set of grid holes, (i.e., translated of $M / 2$ in the $Y$ direction, see Fig. 1). With similar 2D PIV measurements in additional planes, and under a specific set of symmetry hypothesis, it would be possible to estimate kinetic energy budgets along $Z$ directions at specific $X$ and $Y$ values, and go further in the modelling of transfer terms and turbulence production. However, three-dimensional three-component measurements of the velocity fields would ultimately be required to measure the total energy transfer terms, or the actual local viscosity mappings. Being now confirmed that the spatial heterogeneity of oscillatory flow is a possible cause of mean flow generation, and that this heterogeneity is induced by spatial confinements effects, further study of the influence of this confinement (e.g., through the $M / L$ ratio) on the flow would be of great interest.

Open Access This article is distributed under the terms of the Creative Commons Attribution 4.0 International License (http://creativeco mmons.org/licenses/by/4.0/), which permits unrestricted use, distribution, and reproduction in any medium, provided you give appropriate credit to the original author(s) and the source, provide a link to the Creative Commons license, and indicate if changes were made.

\section{References}

Alméras E, Risso F, Roig V, Cazin S, Plais C, Augier F (2015) Mixing by bubble-induced turbulence. J Fluid Mech 776:458-474

Barnard BJS, Sellin RHJ (1969) Grid turbulence in dilute polymer solutions. Nature 222:1160-1162

Bouvard M, Dumas H (1967) Application de la méthode de fil chaud à la mesure de la turbulence dans l'eau. La Houille Blanche 3:257-270

Brumley BH, Jirka GH (1987) Near-surface turbulence in a gridstirred tank. J Fluid Mech 183:235-263

Cagney N, Balabani S (2019) Taylor-Couette flow of shear-thinning fluids. Phys Fluids 31(5):053,102

Chiapponi L, Longo S, Tonelli M (2012) Experimental study on oscillating grid turbulence and free surface fluctuation. Exp Fluids 53(5):1515-1531

Cocconi G, De Angelis E, Frohnapfel B, Baevsky M, Liberzon A (2017) Small scale dynamics of a shearless turbulent/nonturbulent interface in dilute polymer solutions. Phys Fluids 29(7):075,102

Comte-Bellot G, Corrsin S (1966) The use of a contraction to improve the isotropy of grid-generated turbulence. J Fluid Mech 25(4):657-682

Cuthbertson AJS, Samsami F, Dong P (2018) Model studies for flocculation of sand-clay mixtures. Coast Eng 132:13-32

Cuvelier G, Launay B (1986) Concentration regimes in xanthan gum solutions deduced from flow and viscoelastic properties. Carbohydr Polym 6(5):321-333

Dickinson SC, Long RR (1983) Oscillating-grid turbulence including effects of rotation. J Fluid Mech 126:315-333

Drayton MJ (1993) Eulerian and Lagrangian Studies of Inhomogeneous Turbulence Generated by an Oscillating Grid. PhD thesis, King's College, University of Cambridge

Escudié R, Liné A (2003) Experimental analysis of hydrodynamics in a radially agitated tank. AIChE J 49(3):585-603

Fabula AG (1966) An experimental study of grid turbulence in dilute high-polymer solutions. PhD thesis, The Pennsylvania State University, University Park, PA

Friehe CA, Schwarz WH (1970) Grid-generated turbulence in dilute polymer solutions. J Fluid Mech

Gabelle JC, Morchain J, Anne-Archard D, Augier F, Liné A (2013) Experimental determination of the shear rate in a stirred tank with a non-newtonian fluid: Carbopol. AIChE J 59(6):2251-2266

Gabelle JC, Morchain J, Liné A (2017) Kinetic energy transfer between first proper orthogonal decomposition modes in a mixing tank. Chem Eng Tech 40(5):927-937

Garcia-Ochoa F, Santos VE, Casas JA, Gomez E (2000) Xanthan gum: production, recovery, and properties. Biotechnol Adv 18(7):549-579

Greated CA (1969) Effect of polymer additive on grid turbulence. Nature 224(5225):1196-1197

Hearst RJ (2015) Fractal, classical, and active grid turbulence: From production to decay. $\mathrm{PhD}$ thesis, University of Toronto (Canada)

Herlina $\mathrm{H}$ (2005) Gas transfer at the air-water interface in a turbulent flow environment. $\mathrm{PhD}$ thesis, Universitätsverlag Karlsruhe, Karlsruhe

Holdsworth DW, Norley CJD, Frayne R, Steinman DA, Rutt BK (1999) Characterization of common carotid artery blood-flow waveforms in normal human subjects. Physiol Meas 20(3):219

Hopfinger EJ, Toly JA (1976) Spatially decaying turbulence and its relation to mixing across density interfaces. J Fluid Mech 78(01):155-175

Hussain AKMF, Reynolds WC (1970) The mechanics of an organized wave in turbulent shear flow. J Fluid Mech 41(2):241-258 
Janzen JG, Herlina H, Jirka GH, Schulz HE, Gulliver JS (2010) Estimation of mass transfer velocity based on measured turbulence parameters. AIChE J 56(8):2005-2017

Katzbauer B (1998) Properties and applications of xanthan gum. Polym Degrad Stab 59(1):81-84

Lacassagne $\mathrm{T}$ (2018) Oscillating grid turbulence and its influence on gas liquid mass trasnfer and mixing in non-Newtonian media. $\mathrm{PhD}$ Thesis, University of Lyon, INSA Lyon

Lacassagne T, Simoëns S, EL Hajem M, Lyon A, Champagne JY (2019) Oscillating grid turbulence in shear-thinning polymer solutions. Phys Fluids 31(8):083,102

Liberzon A, Holzner M, Lüthi B, Guala M, Kinzelbach W (2009) On turbulent entrainment and dissipation in dilute polymer solutions. Phys Fluids (1994-present) 21(3):035,107

Mahamod MT, Mohtar WHMW, Yusoff SFM (2017) Spatial and temporal behavior of $\mathrm{pb}, \mathrm{cd}$ and $\mathrm{zn}$ release during short term low intensity resuspension events. J Teknologi 80(1)

Massey WT, Harris MC, Deglon DA (2012) The effect of energy input on the flotation of quartz in an oscillating grid flotation cell. Miner Eng 36-38:145-151

McComb WD, Allan J, Greated CA (1977) Effect of polymer additives on the small-scale structure of grid-generated turbulence. Phys Fluids 20(6):873-879

McCorquodale MW, Munro RJ (2017) Experimental study of oscillating-grid turbulence interacting with a solid boundary. J Fluid Mech 813:768-798

McCorquodale MW, Munro RJ (2018a) Analysis of intercomponent energy transfer in the interaction of oscillating-grid turbulence with an impermeable boundary. Phys Fluids 30(1):015,105

McCorquodale MW, Munro RJ (2018b) A method for reducing mean flow in oscillating-grid turbulence. Exp Fluids 59(12):182

Mcdougall TJ (1979) Measurements of turbulence in a zero-mean-shear mixed layer. J Fluid Mech 94(03):409-431

McKenna SP, McGillis WR (2004a) Observations of flow repeatability and secondary circulation in an oscillating grid-stirred tank. Phys Fluids (1994-present) 16(9):3499-3502

McKenna SP, McGillis WR (2004b) The role of free-surface turbulence and surfactants in air-water gas transfer. Int J Heat Mass Transf 47(3):539-553

Mignot E, Cai W, Launay G, Riviere N, Escauriaza C (2016) Coherent turbulent structures at the mixing-interface of a square openchannel lateral cavity. Phys Fluids 28(4):045,104

Nagami Y, Saito T (2013) An experimental study of the modulation of the bubble motion by gas-liquid-phase interaction in oscillatinggrid decaying turbulence. Flow Turbul Combust 92(1-2):147-174

Nguyen MQ, Delache A, Simoëns S, Bos WJT, EL Hajem M (2016) Small scale dynamics of isotropic viscoelastic turbulence. Phys Rev Fluids 1(8):083,301

Nokes RI (1988) On the entrainment rate across a density interface. J Fluid Mech 188:185-204

Petříček R, Moucha T, Rejl FJ, Valenz L, Haidl J (2017) Volumetric mass transfer coefficient in the fermenter agitated by Rushton turbines of various diameters in viscous batch. Int J Heat Mass Transf 115:856-866

Rastello M, Michallet H, Marié JL (2017) Sediment erosion in zeromean-shear turbulence. Coast Dyn 094:597-607

Rodd AB, Dunstan DE, Boger DV (2000) Characterisation of xanthan gum solutions using dynamic light scattering and rheology. Carbohydr Polym 42(2):159-174
Rouse HJ, Dodu J (1955) Turbulent diffusion across a density discontinuity. La Houille Blanche 4:522-532

Safari M, Harris M, Deglon D (2017) The effect of energy input on the flotation of a platinum ore in a pilot-scale oscillating grid flotation cell. Miner Eng 110:69-74

San L, Long T, Liu CCK (2017) Algal bioproductivity in turbulent water: an experimental study. Water 9(5):304

Shy SS, Tang CY, Fann SY (1997) A nearly isotropic turbulence generated by a pair of vibrating grids. Exp Thermal Fluid Sci 14(3):251-262

Silva IPDD, Fernando HJS (1994) Oscillating grids as a source of nearly isotropic turbulence. Phys Fluids (1994-present) 6(7):2455-2464

Simoëns S (1992) Applications de l'analyse d'imagec à des phénomènes de mélange et de dispersion turbulents. $\mathrm{PhD}$ thesis, Ecole Centrale de Lyon

Thompson SM, Turner JS (1975) Mixing across an interface due to turbulence generated by an oscillating grid. J Fluid Mech 67(02):349-368

van Doorn E, White CM, Sreenivasan KR (1999) The decay of grid turbulence in polymer and surfactant solutions. Phys Fluids (1994-present) 11(8):2387-2393

Verso L, van Reeuwijk M, Liberzon A (2017) Steady state model and experiment for an oscillating grid turbulent two-layer stratified flow. Phys Rev Fluids 2(10):104,605

Vonlanthen R, Monkewitz PA (2013) Grid turbulence in dilute polymer solutions: PEO in water. J Fluid Mech 730:76-98

Voropayev SI, Fernando HJS (1996) Propagation of grid turbulence in homogeneous fluids. Phys Fluids 8(9):2435-2440

Wan Mohtar WHM (2016) Oscillating-grid turbulence at large strokes: revisiting the equation of Hopfinger and Toly. J Hydrodyn Ser B 28(3):473-481

Wang Y, Cai WH, Wei TZ, Wang L, Li FC (2015) Experimental Study on Two-Oscillating Grid Turbulence With Polymer Additives. ASME, Seoul, South Korea, p V001T15A008

Wang Y, Cai WH, Wei TZ, Zhang HN, Wang L, Li FC (2016) Proper orthogonal decomposition analysis for two-oscillating grid turbulence with viscoelastic fluids. Adv Mech Eng 8(11): 1687814016679,773

Wyatt NB, Liberatore MW (2009) Rheology and viscosity scaling of the polyelectrolyte xanthan gum. J Appl Polym Sci 114(6):4076-4084

Wyatt NB, Gunther CM, Liberatore MW (2011) Drag reduction effectiveness of dilute and entangled xanthan in turbulent pipe flow. J Nonnewton Fluid Mech 166(1):25-31

Xuequan E, Hopfinger EJ (1986) On mixing across an interface in stably stratified fluid. J Fluid Mech 166:227-244

Zhang JF, Zhang QH, Maa JPY, Gq Qiao (2017) Lattice Boltzmann simulations of oscillating-grid turbulence. J Hydrodyn Ser B 29(1):68-74

Publisher's Note Springer Nature remains neutral with regard to jurisdictional claims in published maps and institutional affiliations. 